\title{
comportement d'interface et prévision du frottement latéral le long des pieux et tirants d'ancrage
}

\author{
interface behaviour and prediction \\ of the lateral shaft friction along piles and anchors
}

\author{
M. BOULON
}

Maître de Conférence à l'I.U.T. I de Grenoble, Chercheur à l'Institut de Mécanique de Grenoble*

\section{PLYTAS}

Chercheur à l'Institut de Mécanique de Grenoble*

\section{P. FORAY}

Maître de Conférence à l'Institut National Polytechnique de Grenoble, Chercheur à l'Institut de Mécanique de Grenoble*

\section{Résumé}

Les auteurs présentent une analyse des phénomènes de frottement à partir d'observation sur les pieux, les tirants d'ancrage et les essais de cisaillement en laboratoire sur divers types de sols pulvérulents, en tant que mécanisme élémentaire. II est proposé une modélisation de ces phénomènes de grands cisaillements localisés sous forme de loi d'interface à dépendance directionnelle. Des applications au calcul du comportement de pieux et de tirants d'ancrage sollicités axialement sont présentées, utilisant la méthode des éléments finis. Enfin, un essai de cisaillement direct spécial, dit à rigidité imposée, est proposé en tant que donnée cohérente à utiliser dans la méthode des coefficients de raideur. Les auteurs concluent sur une discussion comparative de l'ensemble de ces résultats, sur les possibilités de simulation du mode de mise en place, et sur les liens de cette approche avec les essais sur pieux modèles en cellules pressurisées souvent appelées chambres de calibration ou d'étalonnage.

\footnotetext{
Abstract

From observation on piles, anchors and from numerous laboratory direct shear tests involving various granular soils, the authors present an analysis of friction phenomena as elementary mechanism. A modelization of the effects of these localized shear at large amplitude is proposed, within the framework of a directionnally dependent interface constitutive equation. Some applications to the prediction of the behaviour of piles and anchors axially loaded are shown by the finite element method, or by a simplified model. In addition, a new direct shear test at imposed normal stiffness is recommended, as coherent data to be used in the method of the sub-grade reaction coefficient ( $t-z$ curves). At last, the relations between the interface approach and the calibration chamber tests is discussed, after comparing the set of experimental and theoritical results, and appraising the simulation of the installation stage.
} 


\section{INTRODUCTION}

Les pieux sollicités en tension ou en compression, ainsi que les tirants d'ancrage sont désormais un mode privilégié de fixation au sol des ouvrages tant terrestres qu'en haute mer. De même, la capacité à la rupture de ces inclusions et donc les charges de service qu'elles sont amenées à supporter sont couramment améliorées grâce aux techniques de cimentation ou d'injection. C'est pourquoi l'évaluation du frottement latéral prend une importance sans cesse croissante. De plus, l'existence de charges cycliques, qu'elles soient naturelles (houle, ...) artificielles (circulation, machines tournantes, ...) ou accidentelles (séismes, ...) oblige le concepteur à se préoccuper non plus seulement des charges limites classiques, mais aussi de l'ensemble de la réponse du pieu ou du tirant à l'histoire du chargement appliqué, ce que permettent des méthodes telles que la méthode des éléments finis ou la méthode de coefficients de raideur (courbes $p-y$, courbes $t-z$ ), à condition toutefois de faire fonctionner ces modèles mathématiques avec des modèles rhéologiques représentatifs. Cette communication porte plus particulièrement sur la modélisation du frottement latéral entre une inclusion et le sol avoisinant. La première partie est consacrée à l'analyse des phénomènes de frottement en milieu pulvérulent; la seconde partie est orientée vers la modélisation de ce phénomène, grâce à une loi dite d'interface ; la troisième partie comporte des applications numériques et les vérifications expérimentales correspondantes. Enfin, nous terminerons par une étude critique des différences à attendre entre les résultats obtenus par une méthode rigoureuse (éléments finis) et par une méthode simplifiée (coefficients de raideur)

\section{ANALYSE DES PHÉNOMÈNES DE FROTTEMENT EN MILIEU PULVÉRULENT}

Ces phénomènes physiques ont été étudiés à travers un grand nombre d'essais in situ et de laboratoire, conduisant à un faisceau de résultats interprétables, à quelques ambiguités près.

A propos des essais in situ d'arrachement de pieux ou tirants, d'enfoncement de pieux, on parle généralement du coefficient de pression des terres $\mathrm{K}$, rapport entre la contrainte radiale moyenne instantanée censée agir sur l'inclusion, et la contrainte effective verticale initiale due au poids des terres à la même profondeur z ( $\gamma$ 'étant le poids volumique déjaugé du sol) :

$$
K=\frac{\sigma_{\text {rad }}}{\gamma^{\prime} z}
$$

En fait, on mesure très rarement les contraintes radiales en question sur le terrain; on ne mesure que les contraintes de cisaillement mobilisées, si bien que l'accès à $\mathrm{K}$ est indirect, supposant connu le frottement réel instantané sol-inclusion tgo :

$$
\mathrm{K}=\frac{\tau}{\gamma^{\prime} \cdot 2 \cdot \operatorname{tg} \delta}
$$

La difficulté d'interprétation est accrue dans le cas de pieux ou tirants injectés, car la géométrie du contact sol-inclusion est alors mal connue (GABAIX et al. (20)). Cette imprécision mise à part, les valeurs de $\mathrm{K}$ s'étagent entre 0,5 et 2 pour les pieux terrestres courants en sol peu cohérent (COYLE et CASTELLO (8), PUECH (32)). Tous les auteurs s'accordent à penser que $\mathrm{K}$ dépend du mode de mise en place, diminue avec la profondeur, et augmente avec la densité relative ou le degré de surconsolidation. Un certain nombre d'essais mentionnés ci-dessous montrent que le coefficient $\mathrm{K}$ varie durant la sollicitation de l'inclusion; $\operatorname{tg} \delta$ étant peu sujet à de grandes variations pour un contact donné, il est clair que la contrainte normale à l'inclusion, o rad, varie durant la sollicitation.

Le laboratoire fournit une palette très variée d'essais susceptibles d'améliorer notre compréhension des phénomènes : citons en particulier les essais de pieux, tirants et autres modèles en cuve de petite dimension, les essais sur modèle en chambre de calibration, et enfin les essais de cisaillement direct plan ou rotatif d'une plaque rugueuse sur un sol.

Les coefficients $\mathrm{K}$ à la rupture mesurés en laboratoire sur pieux, tirants ou autres modèles de petites dimensions, sont sensiblement plus élevés (3 à 10) qu'in situ ainsi que le montrent les expériences de SCHLOSSER et GUILLOUX (34), WERNICK (36), PUECH, FORAY et al. (31). Ce type d'essai présente un très grand intérêt phénoménologique dans le sens où la mesure directe des contraintes normales est possible (PUECH, FORAY et al. (31)). On peut estimer que le phénomène ainsi mis en évidence est qualitativement le même qu'in situ; seul l'aspect quantitatif est différent, puisque les phénomènes de dilatancecontractance sont évidemment liés au niveau de contrainte. Les essais en semi-grandeur sur matériaux calibrés (sable ou gravier) réalisés par DAVIS et PLUMELLE (10), OSTERMAYER et SCHEELE (29) conduisent, comme on pouvait s'y attendre, à des résultats situés, du point de vue de $K$, entre ceux du laboratoire et ceux des essais réels.

Toutes les expériences précédemment citées concernent des niveaux de contraintes faibles à moyens (inférieurs à $200 \mathrm{kPa}$ ) en terme de contrainte moyenne; les essais de BELOTTI et al. (1), réalisés en chambre de calibration sur pieux modèles de petit diamètre, permettent de saisir les phénomènes intervenant dans le domaine des plus fortes contraintes (200 à $800 \mathrm{kPa}$ ). dont relèvent les pieux des travaux en haute mer: $\mathrm{K}$ diminue lorsque la profondeur augmente et peut atteindre des valeurs inférieures à 0,5 généralement considéré comme un minimum (norme de l'American Petroleum Institute). Ces essais montrent également une influence des conditions aux limites latérales sur la cellule, influence qui s'amenuise lorsque les contraintes (la profondeur) augmentent.

L'ensemble de ces résultats suggère l'étude du mécanisme élémentaire de frottement, grâce à un outil adapté. L'appareil le plus adéquat semble être la boîte de cisaillement direct, dont le principal intérêt est d'induire une rupture très localisée au voisinage de l'inclusion, du type de celle qu'on peut observer le long des pieux et tirants (DAVIS et PLUMELLE (10)). Il 
reste que cet appareil produit un essai hétérogène, interprétable avec des hypothèses restrictives. FEDA (15). (16) a présenté une étude assez exhaustive du frottement matériau sur sol à la boîte de CASA. GRANDE, en précisant que l'essai à volume constant était plus représentatif que l'essai classique à contrainte normale constante. YOSHIMI et KISHIDA (37) font état d'essais très bien instrumentés réalisés à la boîte de cisaillement circulaire, et mettant en évidence le rôle du diamètre des grains, de la rugosité de l'inclusion, et du type d'essai.

Des essais de cisaillement direct plan sable sur plaque rugueuse (fig. 1), à la boîte de CASAGRANDE (boîtes circulaires de diamètres $6 \mathrm{~cm}$ et $10 \mathrm{~cm}$ ) sur trois tranches granulométriques (fig. 2) du sable quartzeux d'Hostun ont été également faits à l'Institut de Mécanique de Grenoble. On a principalement étudié les effets :

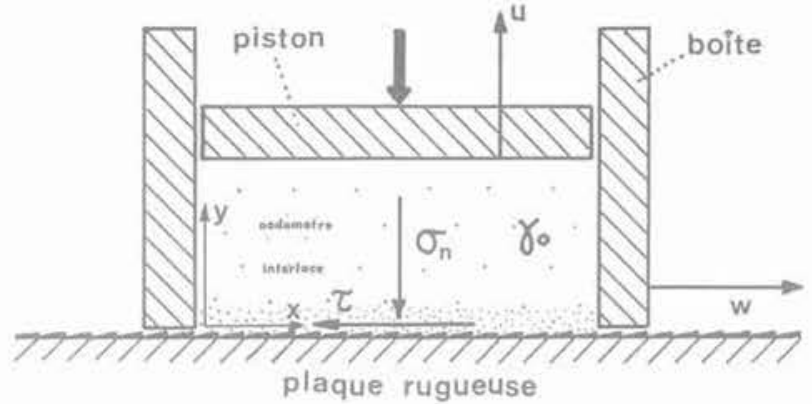

Fig. 1. - Schéma de principe du cisaillement direct plan sable/surface rugueuse et variables de l'essai.

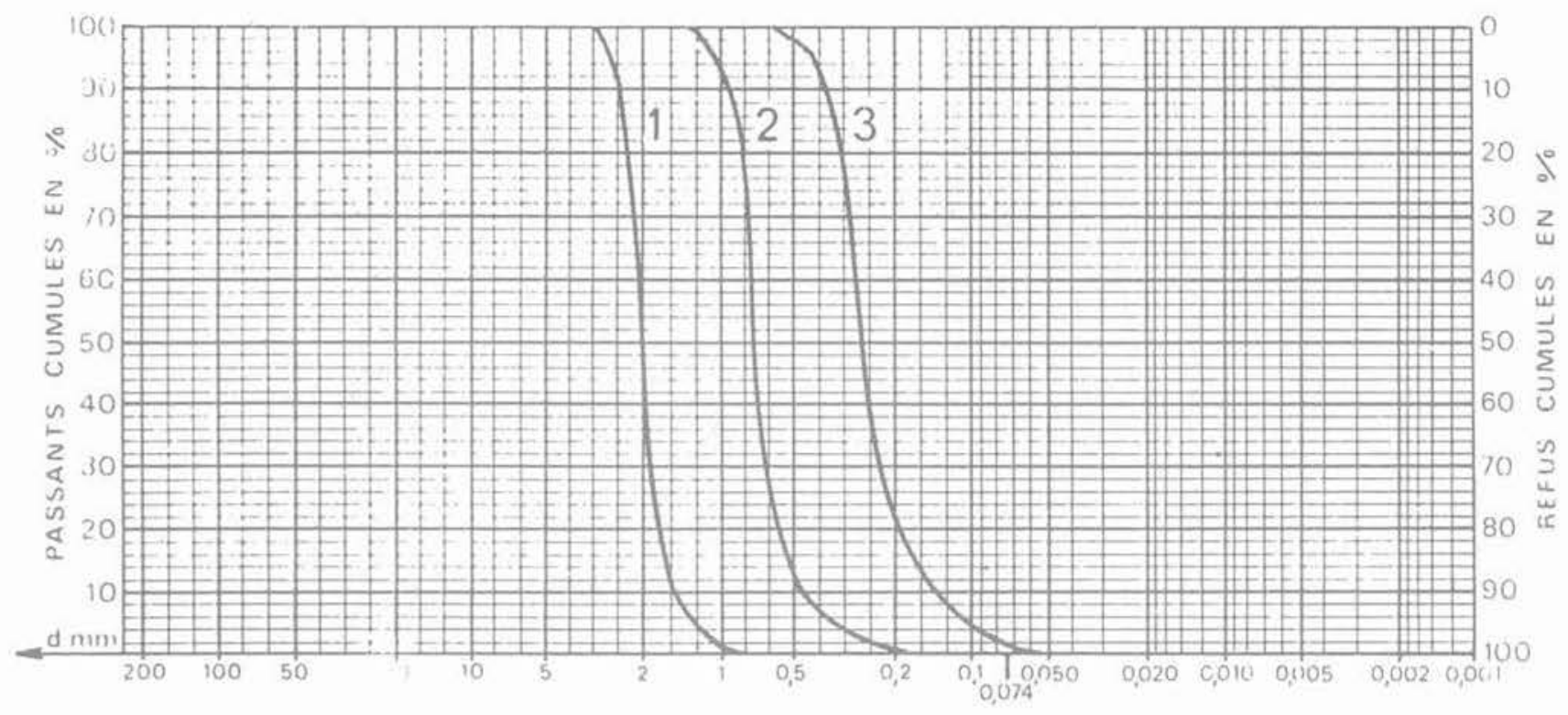

\begin{tabular}{|c|c|c|c|c|}
\hline SABLE & $d_{50}(\mathrm{~mm})$ & $\mathrm{d}_{60} / \mathrm{d}_{10}$ & $\begin{array}{r}\gamma_{\max } \\
\left(\mathrm{kN} \cdot \mathrm{m}^{-3}\right)\end{array}$ & $\left(\mathrm{kN} \cdot \mathrm{m}^{-3}\right)$ \\
\hline 1 & $\# 2.0$ & $\# 1.4$ & $\# 17.8$ & $\# 14.2$ \\
\hline 2 & $\# 0.7$ & $\# 1.7$ & $\# 17.2$ & $\# 13.8$ \\
\hline 3 & $\# 0.32$ & $\# 1.7$ & $\# 16.0$ & $\# 13.2$ \\
\hline
\end{tabular}

Fig. 2. - Caractéristiques physiques des tranches granulométriques du sable d'Hostun utilisé. 

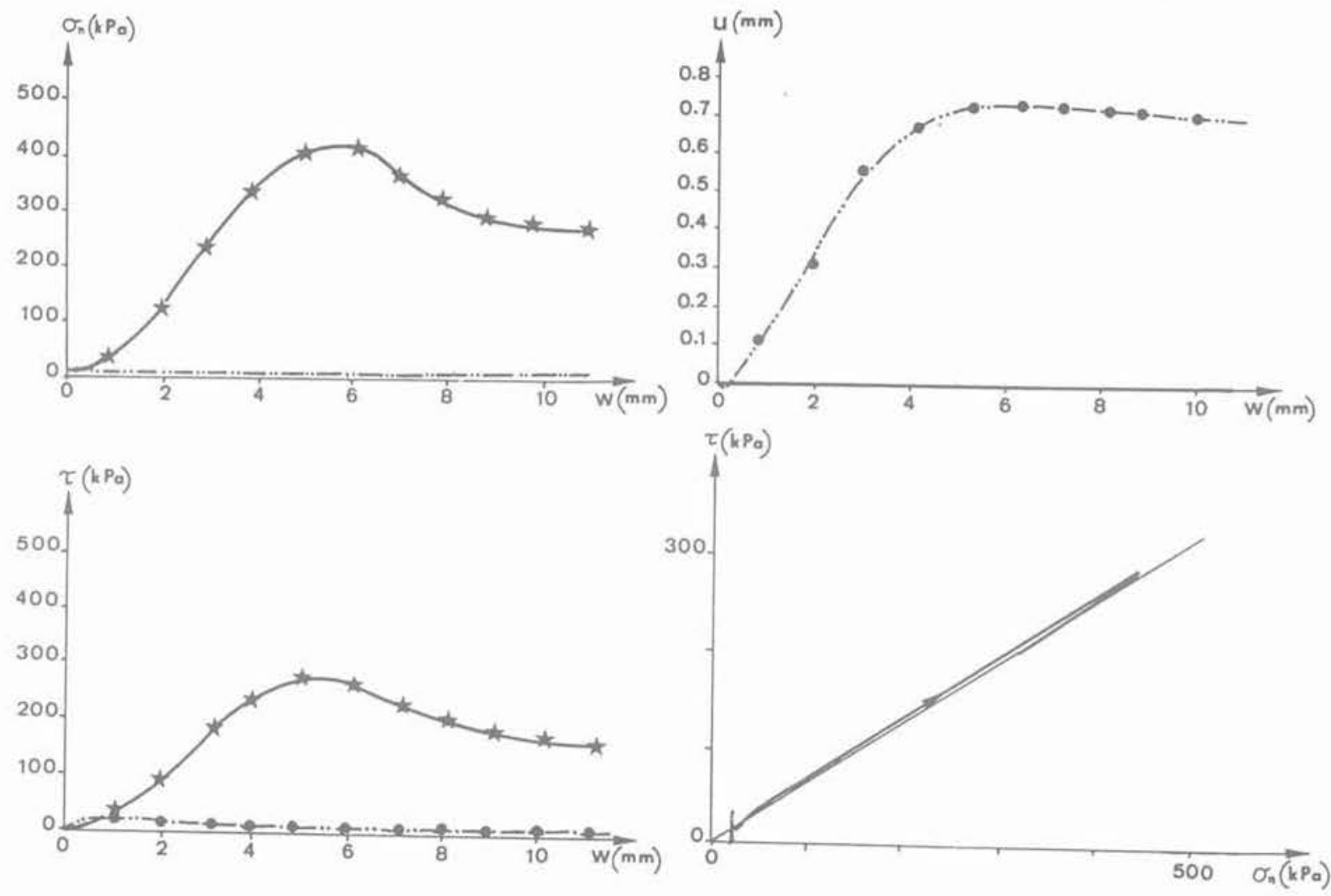

Fig. 3. - Essais à $\sigma_{n}$ constante et à volume constant sur sable d'Hostun n ${ }^{\circ} 2$; $\sigma_{n_{0}}=12,5 \mathrm{kPa} ; \gamma_{0}=16,7 \mathrm{kN} / \mathrm{m}^{3}$ (densité forte)

- Essai à contrainte normale constante.

* Essai à volume constant.
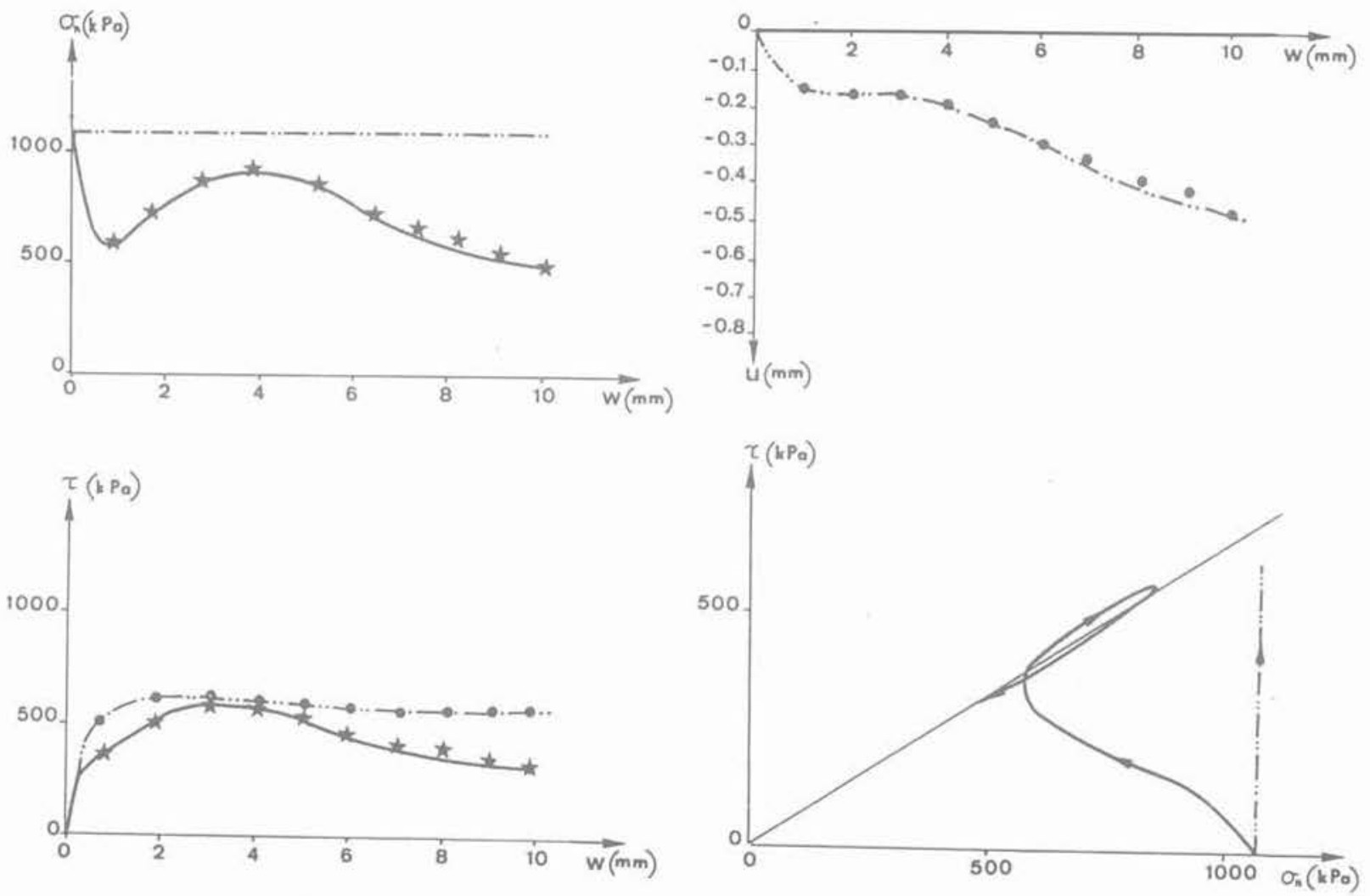

Fig. 4. - Essais à $\sigma_{n}$ constante et à volume constant sur sable d'Hostun n ${ }^{\circ} 2$; $\sigma_{n_{0}}=1415 \mathrm{kPa} ; \gamma_{0}=15,4 \mathrm{kN} / \mathrm{m}^{3}$ (densité faible)

- Essai à contrainte normale constante.

* Essai à volume constant. 
- de la densité initiale;

- de la contrainte normale initiale;

- de la taille des grains;

- du chemin.

Nous donnons seulement ci-dessous quelques résultats typiques et renvoyons à PLYTAS (30) ou BOULON et al. (7) pour plus de détails. Il est intéressant par exemple de comparer les essais de cisaillement direct à contrainte normale constante $(\mathrm{C})$ et à volume constant (V) : pour une contrainte normale initiale faible et une densité initiale forte (fig. 3), l'essaí (V) amplifie au maximum la contrainte normale initiale dans un rapport voisin de 40 , tandis que la contrainte de cisaillement maxima mobilisée est environ trente fois plus importante que lors de l'essai (C). Si les conditions initiales de l'essai sont au contraire une contrainte normale initiale élevée et une densité initiale faible (fig. 4), l'essai (V) réduit la contrainte normale initiale dans un rapport inférieur à 0,5 , tandis que la contrainte de cisaillement mobilisée est également réduite, à saturation du frottement, dans un rapport voisin de 0,5 . La partie finale des courbes présentées aux figures 3 et 4 conduit à échafauder plusieurs hypothèses explicatives sur la décroissance après pic :

- il y a rotation de la boîte;

- il y a "perte de matériau» entre la plaque et la boîte ;

- il y a rupture des grains et production constante de fines durant le cisaillement.

Ces trois hypothèses sont réalistes, mais leur incidence exacte ne peut être évaluée avec un appareillage aussi rudimentaire.

Pour un matériau donné, il y a une certaine équivalence entre contrainte normale initiale et densité initiale, ce qui a amené PLYTAS (30) à formuler le concept de contrainte normale initiale pseudo-critique $\left(\sigma_{n_{\text {epd }}}\right)$ permettant de comparer deux matériaux entre eux, et de décrire presque entièrement le cisaillement par des paramètres sans dimension.

L'influence de la taille des grains est particulièrement évidente sur deux de ces paramètres : le déplacement relatif normal maximum $\mathrm{u}^{+}$lors de l'essai (C) et la contrainte normale maxima $\sigma_{n}^{+}$lors de l'essai (V) (fig. 5). Cet effet a été également mentionné par OSTERMAYER et SCHEELE (29).

Un autre résultat intéressant, qui différencie nettement un chemin de cisaillement direct d'un chemin triaxial de révolution est constitué par «l'absence d'état critique „ (qui conduirait - cf. fig. 6 - à $\sigma_{n}^{+}$indépendant de $\sigma_{n_{0}}$, au sens de FLAVIGNY et al. (17)) dans le premier cas, dû principalement, de notre point de vue, à lintense rupture de grains lors du cisaillement localisé.

Cette étude a été limitée au cas d'une inclusion de rugosité dite «normale», c'est-à-dire que du sable identique à celui de l'échantillon avait été collé sur la plaque rugueuse. Il resterait à évaluer l'effet de la rugosité de la plaque rugueuse (c'est-à-dire de l'inclusion, pieu ou tirant d'ancrage dans la pratique) ainsi que du niveau de résistance à la rupture des grains. En ce qui concerne le sable quartzeux proprement dit, il est clair que le paramètre majeur du cisaillement direct est le chemin, imposé comme nous le verrons dans la suite par les caractéristiques de compressibilité du milieu environnant.

Au terme de cette analyse, nous allons présenter les grands traits d'un modèle de comportement d'interface (les propriétés du contact sol-inclusion) apte à être utilisé dans un calcul de pieu sous effort axial par exemple, quelle que soit la méthode utilisée (éléments finis ou coefficients de raideur)

\section{MODÈLE BIDIMENSIONNEL DE COMPORTEMENT D'INTERFACE}

Les lois d'interface tirent leur source lointaine de l'analyse de stabilité des massifs rocheux et des modèles d'éléments joints, depuis GOODMAN et al. (22), HEUZE et al. (24), ZIENKIEWICZ et al. (38). Ces modèles sont devenus vraiment représentatifs à partir de l'introduction du couplage entre les phénomènes tangentiels et les phénomènes normaux à l'intérieur du joint (GOODMAN et DUBOIS (23), GHABOUSSI et al. (21)). Enfin, les perfectionnements les plus importants ont porté sur la prise en compte de la dilatancecontractance et de la dépendance fonctionnelle du comportement d'interface par rapport au chemin de sollicitation (HEUZE et BARBOUR (25), LEICHNITZ (28). Toutes les publications de mécanique des sols incluant une simulation numérique de comportement d'interface par l'intermédiaire d'éléments joints utilisent des lois d'interface très simplifiées : citons DESAI (11). (12), (13), HOLLOWAY et al. (26), FRANK et al. (19). Nous avons nous-même réalisé des calculs de pieux par la méthode des éléments finis avec interface rigide-plastique - matériau de COULOMB - [BOU. LON et al. (2), (3), (4), (5), (33)] et nous avons constaté que ce modèle rend compte du comportement des pieux terrestres de fiche moyenne (\#20 m), mais ne peut simuler les essais de laboratoire (forte dilatance), ni le comportement des pieux des travaux en haute mer (forte contractance). Sur le plan pratique, nous avons testé plusieurs types de lois pour le sol, depuis l'élasticité linéaire jusqu'à une loi incrémentale octolinéaire (DARVE (9)) : l'influence de la loi du sol proprement dit reste peu importante.

\subsection{Définitions et hypothèses}

Nous désignons par interface la fine zone de sol siège de grands bouleversements de structure et de rupture de grains durant un cisaillement localisé intervenant au contact d'une inclusion dans le sol sollicitée axialement ; supposons avoir isolé cette zone ABCDA'B'C'D' (fig. 7) de masse volumique initiale $\gamma_{\circ}$, en déformation plane parallèlement au plan $(\mathrm{x}, \mathrm{z})$, et en état de contrainte homogène par rapport à $\mathrm{x}$ et $\mathrm{z}$. Une variable surmontée d'un point désignera dans la suite la dérivée temporelle de cette variable. 

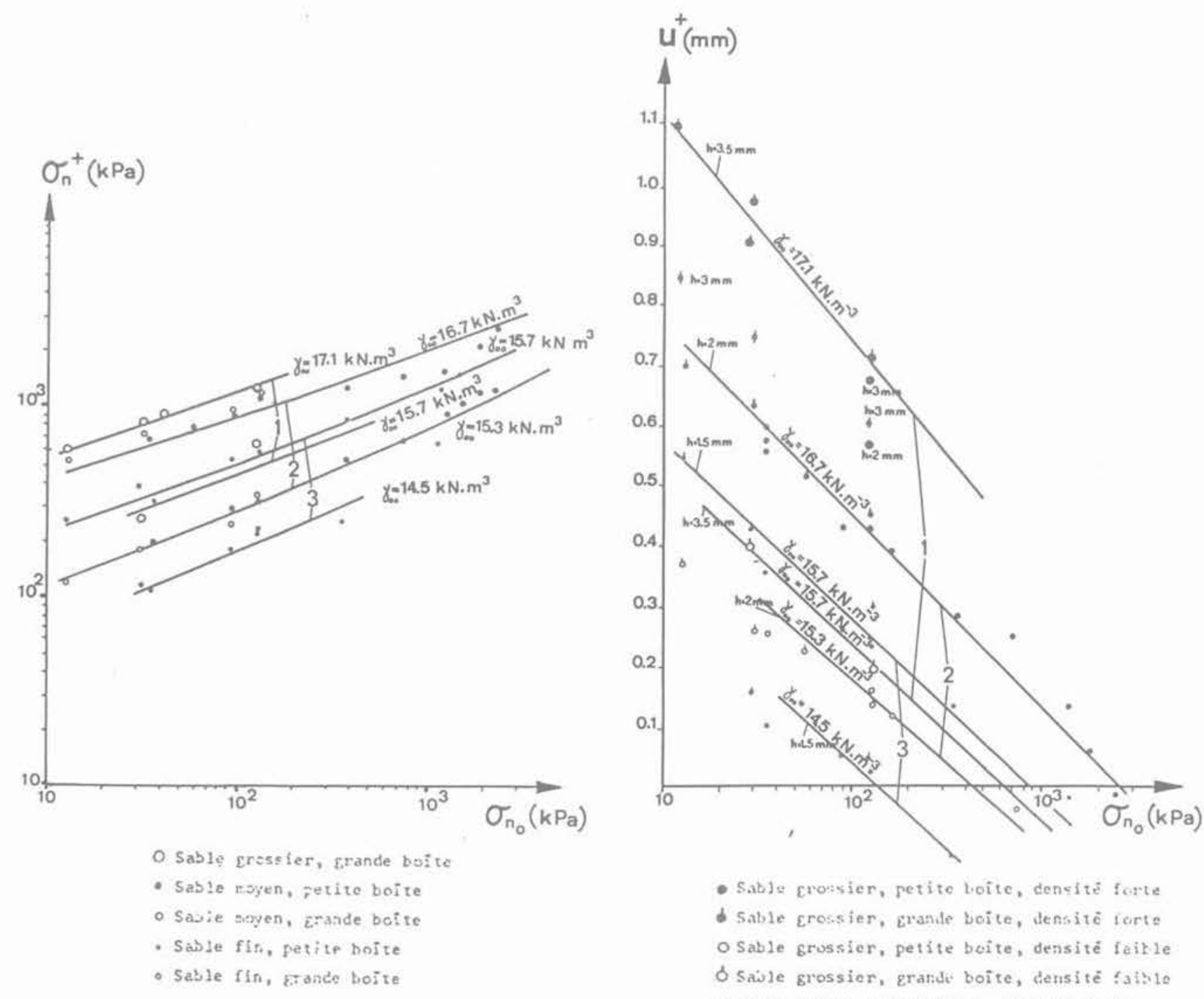

- Sable crousier, petite loite, dencité forte

d Sable Erossier, grande boite, densité ferte

o sable grussier, petite beîte, densité fairle

¿ saijle grossier, grandi boite, densitẻ faiłle

- Sable moyen, petite boite, densité fcrte

- Sable noyen, grande boite, densite forte

- Sable moyen, perite boite, censité faible

- Sable moyen, grande boite, densité faible

- Sable fin, petite buite, densité forte

- Sable fin, erande boile, densité forte

- Sable fin, petite boîte, censité faible

d Sable fin, grande boĩte, densité faible

Fig. 5. - Influence de la taille des grains sur les paramètres $u^{*}$ (déplacement relatif normal maximum lors d'un essai à $\sigma_{n}$ constante) et $\sigma_{n}^{+}$(contrainte normale maxima lors d'un essai à volume constant). 


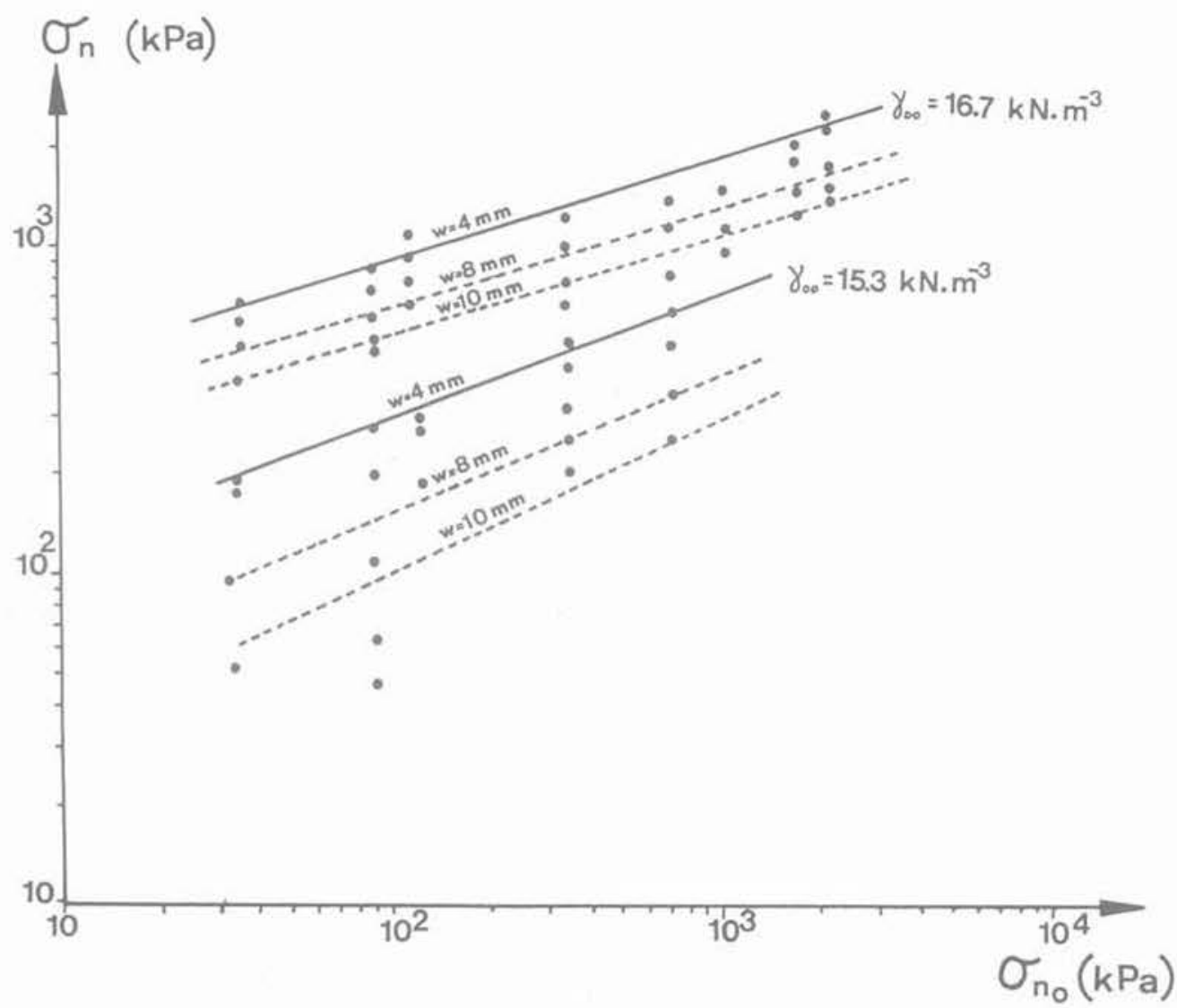

Fig. 6. - Cisaillement direct selon un chemin à volume constant sur sable d'Hostun $n^{\circ} 2: \sigma_{n}$ ne tend pas vers une limite indépendante de $\sigma_{n_{0}}$.

Soient :

$$
\underline{u}=\left\{\begin{array}{c}
w \\
u
\end{array}\right\} \quad \text { et } \quad \underline{\sigma}=\left\{\begin{array}{c}
\tau \\
\sigma_{n}
\end{array}\right\}
$$

respectivement le vecteur déplacement relatif d'interface et le vecteur contrainte agissant sur linterface (composantes écrites dans l'ordre $\mathrm{z}, \mathrm{x}$; composante nulle sur $y$ ) ; le qualificatif «bidimensionnel " indique que $\sigma, \dot{\sigma}$, u et $\dot{u}$ sont et restent coplanaires. $\underline{\sigma}$ est arbitrairement désigné comme sollicitation, et $u$ comme réponse. La loi d'interface complète est supposée être une relation fonctionnelle entre les diverses variables et leurs valeurs initiales :

$$
\mathcal{F}\left(u, \sigma, u_{0}, \sigma_{0}, \gamma_{0}, \ldots\right)=0
$$

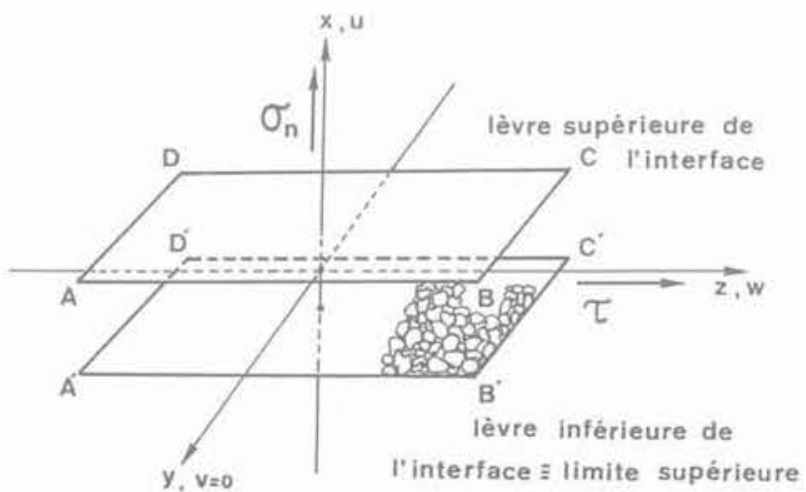

de $\mathrm{I}$ 'inclusion

La dépendance directionnelle de la réponse est obtenue à travers l'expression d'une loi tangente, particulièrement adaptée aux calculs incrémentaux non linéaires. Le chemin tangent est donc repéré en variables réduites; on peut aussi dire qu'il est normé. On sup-

Fig. 7. - Interface; définition géométrique et définition des variables. 
pose la loi tangente non visqueuse et homogène de degré 1 en vitesses de contraintes; posons :

$$
\begin{aligned}
& \dot{\sigma}=\left(\dot{\tau}^{2}+\dot{\sigma}_{n}^{2}\right)^{1 / 2} \quad \dot{\sigma} \geqslant 0 \\
& \xi=\frac{\dot{\tau}}{\sigma}-1 \leqslant \xi, \eta \leqslant 1 \\
& \eta=\frac{\dot{\sigma}_{n}}{\dot{\sigma}} \xi^{2}+\eta^{2}=1
\end{aligned}
$$

Le caractère homogène de degré 1 en $\dot{\sigma}$ rend en fait cette loi « indépendante * de la grandeur de $\dot{\sigma}$, c'est-àdire que le chemin est défini par 1 paramètre ( 2 variables, $\xi$ et $\eta$, liées par une relation). Dans ces conditions, une sollicitation tangente correspond à 1 point du cercle de rayon unité de l'espace $(\xi, \eta)$ (fig. 8.).

\section{2. Élément rhéologique d'interface}

Nous cherchons une interpolation Wi sur les chemins élémentaires en nombre quelconque $\mathrm{n}$ :

sollicitation tangente élémentaire :

$$
\left\{\begin{array}{c}
\dot{\bar{\sigma}} \\
\dot{\sigma}_{\mathrm{n}}
\end{array}\right\}=\left\{\begin{array}{c}
\bar{z}_{1} \\
\eta_{1}
\end{array}\right\} \dot{\sigma} \quad \mathrm{i}=(1, \ldots, \mathrm{n})
$$

réponse tangente élémentaire :

$$
\left\{\begin{array}{l}
\dot{w} \\
u
\end{array}\right\}=\left\{\begin{array}{l}
i_{i} \\
\mu_{i}
\end{array}\right\} \dot{j} \quad i=(1, \ldots, n)
$$

chemin élémentaire $\mathrm{n}^{\circ} \mathrm{i}$

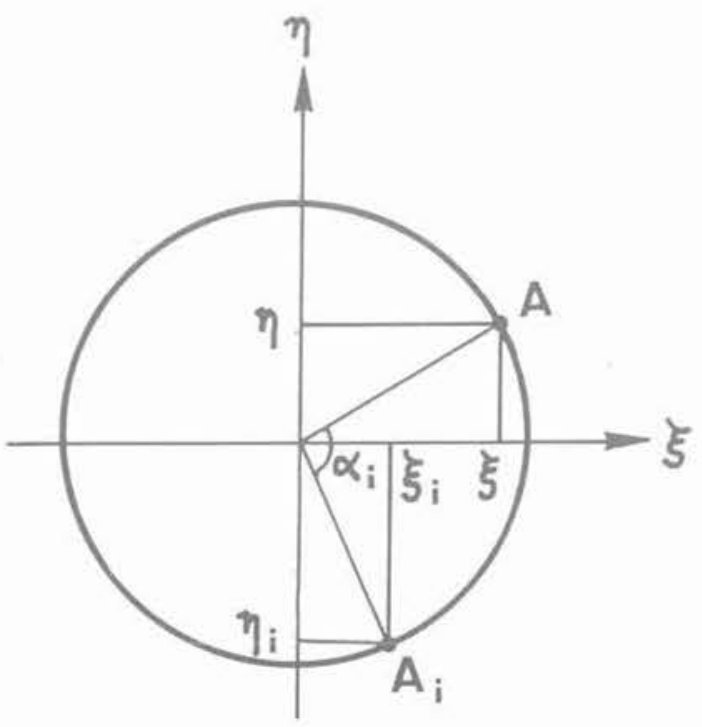

telle que la réponse sur un chemin quelconque soit obtenue ainsi (fig. 8)

sollicitation tangente sur chemin quelconque :

$$
\left\{\begin{array}{c}
\dot{\tau}^{\prime} \\
\dot{\sigma}_{n}
\end{array}\right\}=\left\{\begin{array}{c}
\bar{z} \\
i
\end{array}\right\} \dot{\sigma}
$$

réponse tangente sur chemin quelconque :

$$
\left\{\begin{array}{c}
\dot{w} \\
u
\end{array}\right\}=\sum_{i=1}^{n} \mathrm{~W}_{i}\left\{\begin{array}{l}
\dot{w} \\
u
\end{array}\right\}=\sum_{i=1}^{n} \mathrm{w}_{i}\left\{\begin{array}{l}
i_{i} \\
\mu_{i}
\end{array}\right\} \dot{\sigma}=\left\{\begin{array}{l}
\dot{i} \\
\mu
\end{array}\right\} \dot{\sigma}
$$

La pondération $W_{1}$ est définie par la proximité relative, en termes de distance angulaire, du chemin $(\xi, \eta)$ par rapport aux divers chemins de base $(\xi 1, \eta j)$ (BOULON (6)) à partir des relations suivantes :

$$
\begin{aligned}
& \sum_{i=1}^{n} W_{1}=1 \\
& W_{1} \alpha_{1}^{x}=W_{2} \alpha_{2}^{x}=\ldots=W_{n} \alpha_{n}^{x} \\
& x \in \mathbb{R}^{+}
\end{aligned}
$$

$\mathrm{x}$ caractérise la courbure des fonctions d'interpolation.

Cette interpolation est particulièrement intéressante dans la mesure où elle permet une évaluation facile de l'incertitude provenant des incertitudes expérimentales, ainsi qu'une étude simple de l'unicité de la réponse. Rappelons par ailleurs qu'elle "accepte» un nombre quelconque de chemins élémentaires.

Fig. 8a. - Espace des sollicitations tangentes.

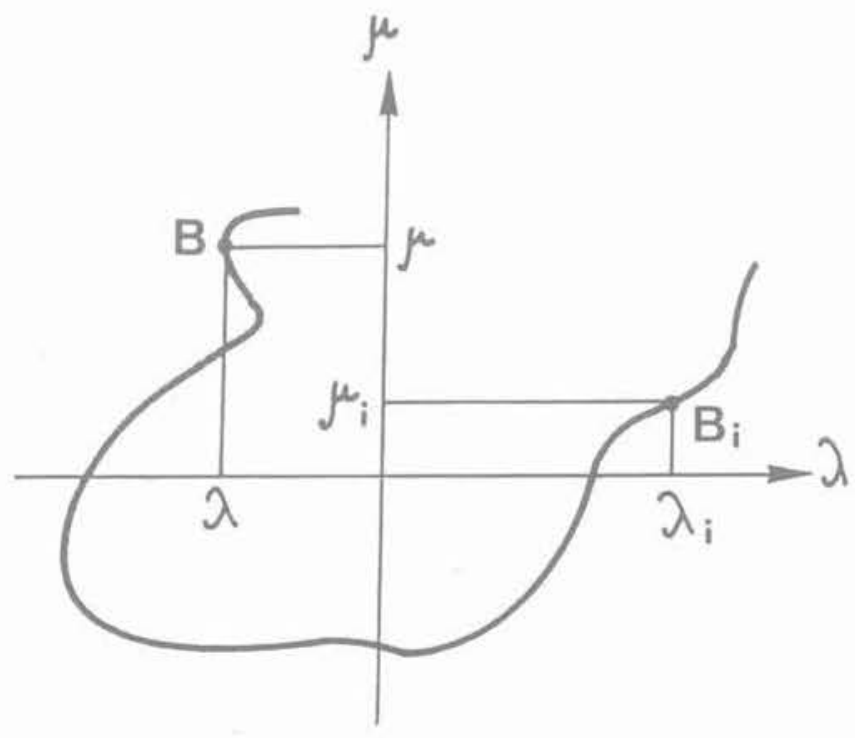

Fig. 8b. - Espace des réponses tangentes. 


\subsection{Identification}

Nous supposons en première approximation que la mémoire du matériau peut être décrite par le poids volumique actuel au sein de l'interface ainsi que $u$ et $\sigma$. Ceci est très schématique, puisque les paramètres tels que la granulométrie, la structure de l'assemblage des grains, ... interviennent également. Par ailleurs, l'essai utilisé est l'essai de cisaillement direct qui, à l'évidence, ne permet pas d' $\propto$ isoler » un interface; nous considérerons toutefois que l'erreur systématique expérimentale reste faible, dans la mesure où un échantillon testé à la boîte de Casagrande est constitué d'une partie "active» (l'interface proprement dit) et d'une partie «inerte» fonctionnant à la manière d'un cedomètre (fig. 1). Nous négligeons donc la compressibilité œedométrique de la partie « inerte » de l'échantillon.

L'identification de la loi est réalisée à partir de chemins de cisaillement a $(\xi i, \eta i)$ fixes ou variables grâce à des tableaux de résultats, éventuellement approchés par des formules, du type :

$$
\left(\lambda_{i}, \mu_{i}\right)=\mathrm{f}\left(\xi_{i}, \eta_{i}, \gamma_{0}, \mathrm{w}, \mathrm{u}, \tau, \sigma_{\mathrm{n}}\right)
$$

Les chemins aisément accessibles à l'expérience de cisaillement sont :

- le chemin à volume constant :

$$
\begin{gathered}
\lambda_{u}=\frac{\partial \dot{w}}{\dot{\partial} \tau_{u}} \cdot \xi_{u}+\frac{\partial \dot{w}}{\partial \dot{\sigma}_{n}} \cdot \eta_{u} \\
\mu_{u}=\frac{\partial \dot{u}}{\partial \dot{\tau}_{u}} \cdot \xi_{u}+\frac{\partial \dot{\mathrm{u}}}{\partial \dot{\sigma}_{n}} \cdot \eta_{u}=0
\end{gathered}
$$

- le chemin à contrainte normale constante ;

$$
\begin{aligned}
& \lambda_{o_{n}}=\frac{\partial \dot{\mathrm{w}}}{\partial \tau} \sigma_{\sigma_{n}} \cdot \xi_{o_{n}} \\
& \mu_{o_{n}}=\frac{\partial \dot{\mathrm{u}}}{\partial \dot{\tau}_{o_{n}}} \cdot \xi_{o_{n}}
\end{aligned} \quad \eta_{o_{n}}=0
$$

Les limites de validité du modèle découlent de la plage de valeurs encadrées par les variables d'identification $\left(\xi_{i}, \eta_{i}, \lambda_{i}, \mu_{i}, \gamma_{0}, \ldots\right)$.

L'utilisation sous forme de loi rhéologique d'interface proprement dite nécessite une linéarisation au voisinage du chemin courant c, exprimée par (12), grâce au théorème d'Euler sur les fonctions homogènes (BOULON (6)) :

$$
\left\{\begin{array}{l}
\dot{w} \\
\dot{u}
\end{array}\right\}=\left[\begin{array}{lll}
\frac{\partial \dot{w}}{\partial \dot{\tau}^{\prime}} & c & \frac{\partial \dot{w}}{\partial \dot{\sigma}_{n}} \\
\frac{\partial \dot{u}}{\partial \dot{\tau}_{c}} & \frac{\partial \dot{u}^{\prime}}{\partial \dot{\sigma}_{n_{c}}}
\end{array}\right] \times\left\{\begin{array}{l}
\dot{\tau}_{n} \\
\dot{\sigma}_{n}
\end{array}\right\}
$$

\section{APPLICATION A LA PRÉVISION DU FROTTEMENT LATÉRAL}

Deux types d'applications sont présentées ici, concernant le frottement latéral le long des pieux moulés. Le premier type est une simulation par la méthode des éléments finis de l'arrachement d'un pieu modèle de Laboratoire; le second type est une application simplifiée des comportements d'interface à la prévision du coefficient $\mathrm{K}$ limite de pression des terres dans un matériau donné, par une simple intégration numérique de la loi d'interface sur un chemin de cisaillement précisé, sans la méthode des éléments finis.

\subsection{Simulation du comportement d'un pieu modèle de Laboratoire par la méthode des éléments finis}

Il s'agit de deux essais d'arrachement d'un pieu modèle de Laboratoire de diamètre égal à $5,5 \mathrm{~cm}$ dit "moulé * c'est-à-dire en fait installé avant déversement de sable dans son voisinage, dont le fut a été rendu rugueux par collage de grains de sable. Ce pieu a été équipé de capteurs permettant d'évaluer les contraintes de cisaillement mobilisées localement, ainsi que les contraintes normales (PUECH, FORAY et al. (31)). L'évolution du vecteur contrainte agissant sur le fût a été mesurée à une profondeur égale à̀ $1,2 \mathrm{~m}$ (le pieu a une fiche de $1,6 \mathrm{~m}$, la cuve a une profondeur de $2 \mathrm{~m}$ et un diamètre de $1,5 \mathrm{~m}$ ).

Le modèle numérique est un modèle aux éléments finis en déplacements mis en œuvre à partir d'un principe des puissances virtuelles adapté à la résolution des problèmes de contact avec frottement (BOULON (6)) :

$$
\begin{aligned}
& \int \dot{\sigma}_{\Omega} \cdot \delta \dot{\varepsilon}_{i j} \cdot d t \cdot d \Omega+\int \dot{t}_{r_{s g}} \cdot\left(\delta \dot{u}_{2}-\delta \dot{u}_{11}\right) \cdot d t \cdot d \Omega \\
& =\int \dot{\mathrm{f}}_{\mathrm{i}} \cdot \delta \dot{\mathrm{u}} \cdot \mathrm{dt} \cdot \mathrm{d} \Omega+\int \dot{\mathrm{t}}_{\mathrm{r}_{a}} \cdot \delta \dot{\mathrm{u}}_{1} \cdot \mathrm{dt} \cdot \mathrm{d} \Gamma \\
& +\int_{\mathrm{dr}_{59}}^{\mathrm{t}_{1 \mathrm{~s}}}\left(\delta \dot{\mathrm{u}}_{1 i}-\delta \dot{\mathrm{u}}_{2 \mathrm{i}}\right) \cdot \mathrm{d} \Gamma \\
& \forall \delta \mathrm{u}, \mathrm{c} . \mathrm{a} .
\end{aligned}
$$

les quantités intervenant dans ce principe, écrit en vue d'un traitement incrémental, sont définies à la figure 9. Le problème est traité en axisymétrie.

La discrétisation spatiale du sol comporte quatre-vingt couples d'éléments triangulaires à trois nœeuds et cent dix nœuds; celle du pieu consiste en six éléments poutre soumis à un effort normal. Nous n'avons pas utilisé d'éléments joints, mais plutôt une approche par sousstructures permettant de contourner les obstacles purement numériques.

Les paramètres mécaniques adoptés pour le sol (élasticité non linéaire) et pour l'interface (voir § 2) sont répertoriés dans le tableau I ci-dessous. 
Tableau 1: Simulation numérique par la méthode des éléments finis; paramètres mécaniques adoptés pour le sol et l'interface (sable d'Hostun n' 2 -pieu moulé).

\begin{tabular}{|c|c|c|c|}
\hline & paramètres & densité forte & densité faible \\
\hline \multirow{6}{*}{$\begin{array}{l}\text { sol (élasticité non linéaire), } \\
\text { d'après LABANIEH (27) }\end{array}$} & poids volumique $\left(\mathrm{kN} / \mathrm{m}^{3}\right)$ & 16,7 & 15,4 \\
\hline & $\begin{array}{l}\mathrm{E}_{100}(\mathrm{MPa}) \text { : module de référence } \\
\text { sous } 100 \mathrm{kPa}\end{array}$ & 53,5 & 30,2 \\
\hline & $\begin{array}{l}n \text { : exposant caractéristique de } \\
\text { variation des modules }\end{array}$ & 0,6 & 0,6 \\
\hline & $\varnothing$ : angle de frottement & $45^{\circ}$ & $45^{\circ}$ \\
\hline & $\begin{array}{l}\text { Ko coefficient de pression des ter- } \\
\text { res au repos }\end{array}$ & 0,43 & 0,43 \\
\hline & $\begin{array}{l}\mathrm{E}_{\text {p100 }}(\mathrm{MPa}) \text { : module pressiométri- } \\
\text { que calculé sous } \sigma_{v_{o}}=100 \mathrm{kPa}\end{array}$ & 54 & 30,5 \\
\hline \multirow{8}{*}{$\begin{array}{l}\text { interface, d'après PLYTAS } \\
(30)\end{array}$} & $\begin{array}{l}\sigma_{\text {no }}(\mathrm{kPa}) \text { : contrainte normale ini- } \\
\text { tiale }\end{array}$ & 12,5 & 12,5 \\
\hline & $\begin{array}{l}\mathrm{u}^{+}(\mathrm{mm}) \text { : déplacement normal } \\
\text { maximal à } \sigma_{n} \text { constante }\end{array}$ & 0,70 & 0,30 \\
\hline & $\begin{array}{l}\sigma_{n}^{+}(\mathrm{kPa}) \text { : contrainte normale maxi- } \\
\text { male à volume constant }\end{array}$ & 500 & 113 \\
\hline & $\delta:$ angle de frottement sol-pieu & $39^{\circ}$ & $39^{\circ}$ \\
\hline & $\left.\frac{\mathrm{d} \tau}{\mathrm{dw}} \quad \mathrm{w}=0, \quad \sigma_{\mathrm{n}}=\mathrm{kPa} / \mathrm{mm}\right)$ & 120 & 39 \\
\hline & $\frac{d \tau}{d w} \quad w=0, \quad \stackrel{(k P a / m m)}{V=C t e}$ & 120 & 28 \\
\hline & $\frac{d u}{d w} \quad w=0, \sigma_{n}=C$ te & $-0,1$ & $-0,1$ \\
\hline & $\frac{\mathrm{d} \sigma_{\mathrm{n}}}{\mathrm{dw}} \quad \mathrm{w}=0, \quad \stackrel{(\mathrm{kPa} / \mathrm{mm})}{V=C t e}$ & -20 & -12 \\
\hline
\end{tabular}

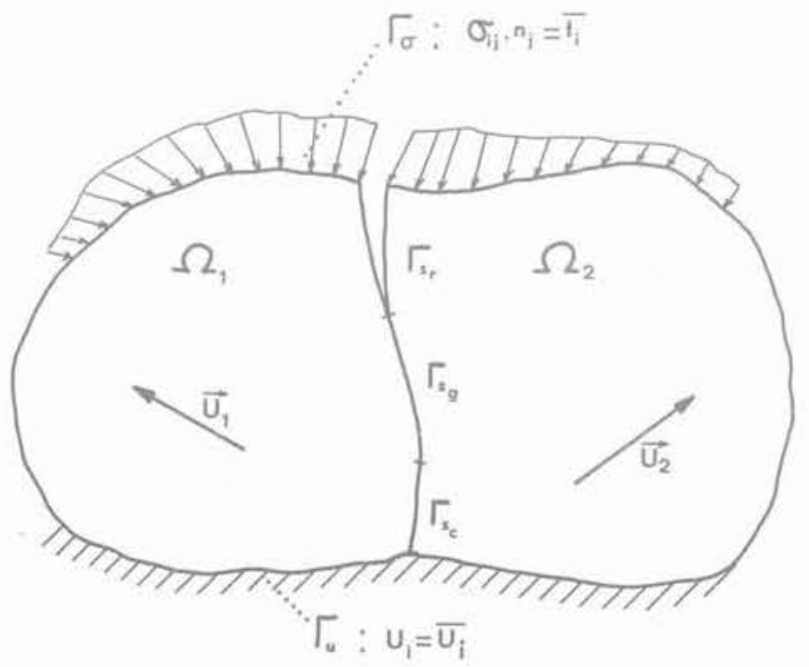

Fig. 9. - Principe des puissances virtuelles adapté à la résolution des problèmes de contact avec glissement relatif, d'après BOULON (6)

Les non-linéarités ont été résolues par la méthode classique des contraintes initiales; quinze pas de chargement ont été nécessaires.

Les résultats numériques obtenus sont présentés aux figures 10 et 11 , et comparés aux résultats expérimentaux correspondants. Les décroissances après pic n'étant pas modélisées dans notre loi d'interface, on aboutit à un palier parfait de $\mathrm{K}$ à 3,4 en densité forte ( 3,8 pour l'expérience) et 1,3 en densité faible (1,5 pour l'expérience). Les augmentations très importantes de contraintes ainsi enregistrées sont évidemment dues aux faibles contraintes normales initiales $(\# 10 \mathrm{kPa}$ ). 


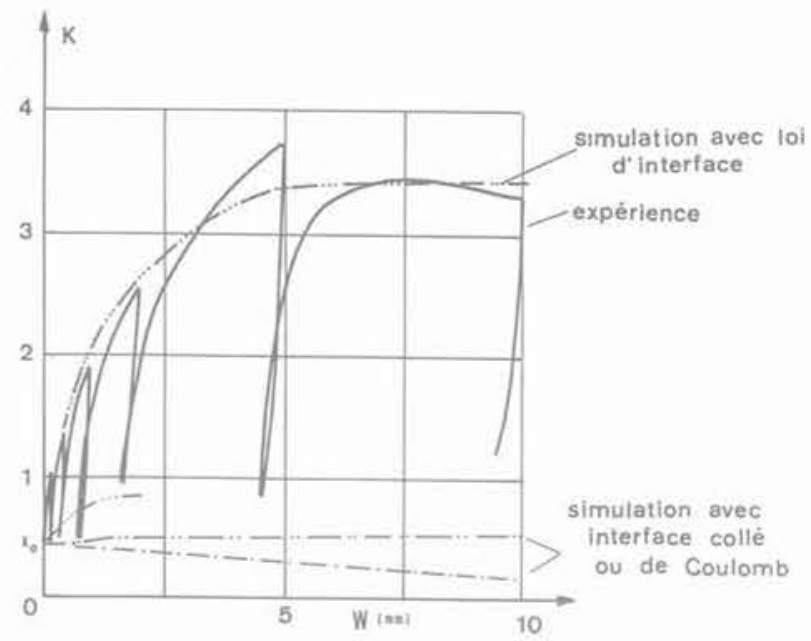

Fig. 10. - Essai d'arrachement de pieu modèle en cuve à sable (Hostun $n^{\circ} 2$ ), expérience et simulation numérique. Densité forte; évolution de $K$ à la profondeur $z=1,2 \mathrm{~m}$.

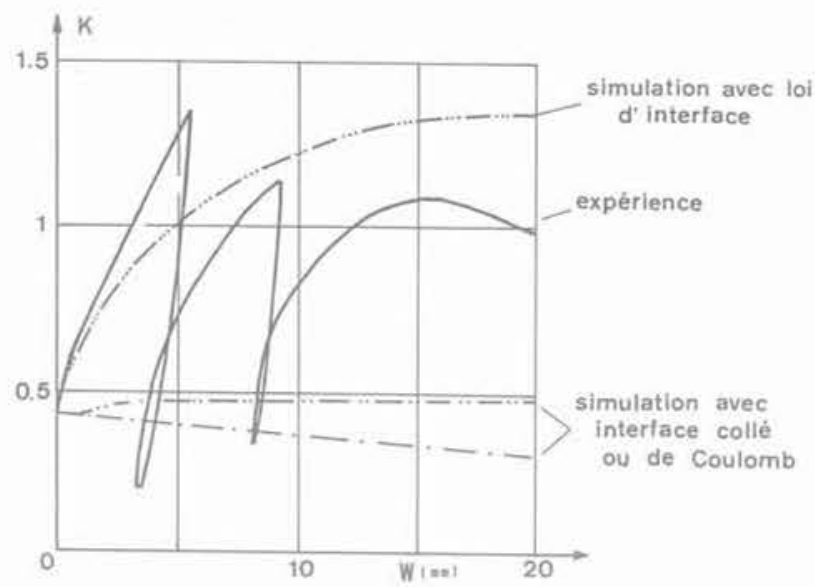

Fig. 11. - Essai d'arrachement de pieu modèle en cuve à sable (Hostun $n^{\circ} 2$ ) expérience et simulation numérique. Densité faible; évolution de $K$ à la profondeur $z=1,2 \mathrm{~m}$.

t temps; dt élément infinitésimal de temps;

$\Omega_{3}=$ sol

$\Omega_{2}=$ pieu

$\Omega=\Omega_{1} \cup \Omega_{2}$

$\Gamma_{u}=\Gamma_{\mathrm{u} 1} \cup \Gamma_{\mathrm{u} 2}$ frontière : conditions aux limites en déplacements : $\dot{u}_{i}=\dot{\bar{u}}_{i}$

$\Gamma \sigma=\Gamma \sigma_{1} \cup \Gamma \sigma_{2}$ frontière : conditions aux limites « en contraintes $s: \dot{t}_{1}=\dot{\sigma}_{i j}, n_{1}=\dot{t}_{i}$

$\Gamma_{S}=\Gamma_{S_{t}} \cup \Gamma_{S_{g}} \cup \Gamma_{S_{c}}=$ interface entre $\Omega_{1}$ et $\Omega_{2}$

$\Gamma_{S_{t}}=$ partie de l'interface : contact rompu antérieurement au temps $t$

$\Gamma_{S_{\mathrm{S}}}=$ partie de l'interface $:$ contact glissant au temps $\mathrm{t}$ $\Gamma_{\mathrm{S}_{c}}=$ partie de l'interface : contact collé au temps $\mathrm{t}$

$\delta \dot{u}_{i}, \delta \dot{\varepsilon}_{i j}=$ champ de vitesse de déplacement virtuel cinématiquement admissible au temps $t$, champ de déformation associé. $\sigma_{i}=$ champ de vitesse de contrainte réel au temps $t$ $\mathrm{f}_{\mathrm{i}}=$ champ de vitesse de forces de volume au temps $\mathrm{t}$.

\subsection{Prévision du coefficient $\mathrm{K}_{\text {lim }}$ de pression des terres le long d'une inclusion «moulée»}

\subsubsection{Intérêt de lessai de cisaillement} à rigidité normale imposée

De même que la prévision des tassements d'une fondation est couramment réalisée par simulation physique approchée du chemin suivi par un volume élémentaire de sol (méthode œedométrique, méthode triaxiale,...). on peut imaginer que la simulation physique approchée du chemin suivi par un volume élémentaire d'interface conduira à une prévision approchée du frottement latéral.

Nous nous limitons pour cela au cas d'une inclusion rigide «moulée» (voir 3.1) latéralement rigide, sollicitée axialement. Si une dilatance ou une contractance très localisée au voisinage de l'inclusion intervient, le sol situé au-delà de la fine zone de transition constituée par l'interface subit en première approximation une sollicitation pressiométrique, à laquelle vient s'ajouter un cisaillement simple dont le niveau décroît très rapidement avec la distance à partir du fut de l'inclusion (FRANK (18)). Si nous négligeons les variations de volume dues à ce cisaillement simple, nous parvenons ainsi à l'«image » d'un cisaillement direct entre l'inclusion et le sol réagissant comme s'il était sollicité par un pressiomètre.

Soient : $\mathrm{R}$, le rayon nominal de l'inclusion (fig. 12); e l'épaisseur initiale de l'interface $\left(e<<R_{0}\right)$; $\mathrm{E}_{\mathrm{p}}$ le module pressiométrique du sol.

Après un déplacement relatif axial local $w$ de l'inclusion par rapport au sol, soient : $\sigma_{n}$ la contrainte normale locale, $\mathrm{u}$ la variation d'épaisseur de l'interface ( $\mathrm{u}<<\mathrm{e}$ ), entre l'état au repos $\left(\mathrm{K}_{\mathrm{o}}\right)$ et un état correspondant au déplacement relatif tangentiel local w l'équilibre statique radial et la comptabilité cinématique s'écrivent:

$$
\frac{\sigma_{n}-\sigma_{n 0}}{\frac{\Delta V}{V}}=E_{p}
$$

avec

$$
\mathrm{V}_{0}=\pi\left(\mathrm{R}_{0}+\mathrm{e}\right)^{2} \# \pi \mathrm{R}^{2}
$$$$
\Delta \mathrm{V} \# 2 \pi\left(\mathrm{R}_{\mathrm{o}}+\mathrm{e}\right) \mathrm{u} \# 2 \pi \mathrm{R}_{\mathrm{o}} \mathrm{u}
$$

d'où

$$
\frac{\sigma_{n}-\sigma_{n_{0}}}{u}=\frac{2 \cdot E_{p}}{R_{0}}
$$

et en raison de la linéarité de la courbe pressiométrique dans la zone des petits déplacements radiaux,

$$
\frac{\sigma_{n}}{u}=\frac{2 \cdot E_{p}}{R_{0}}
$$



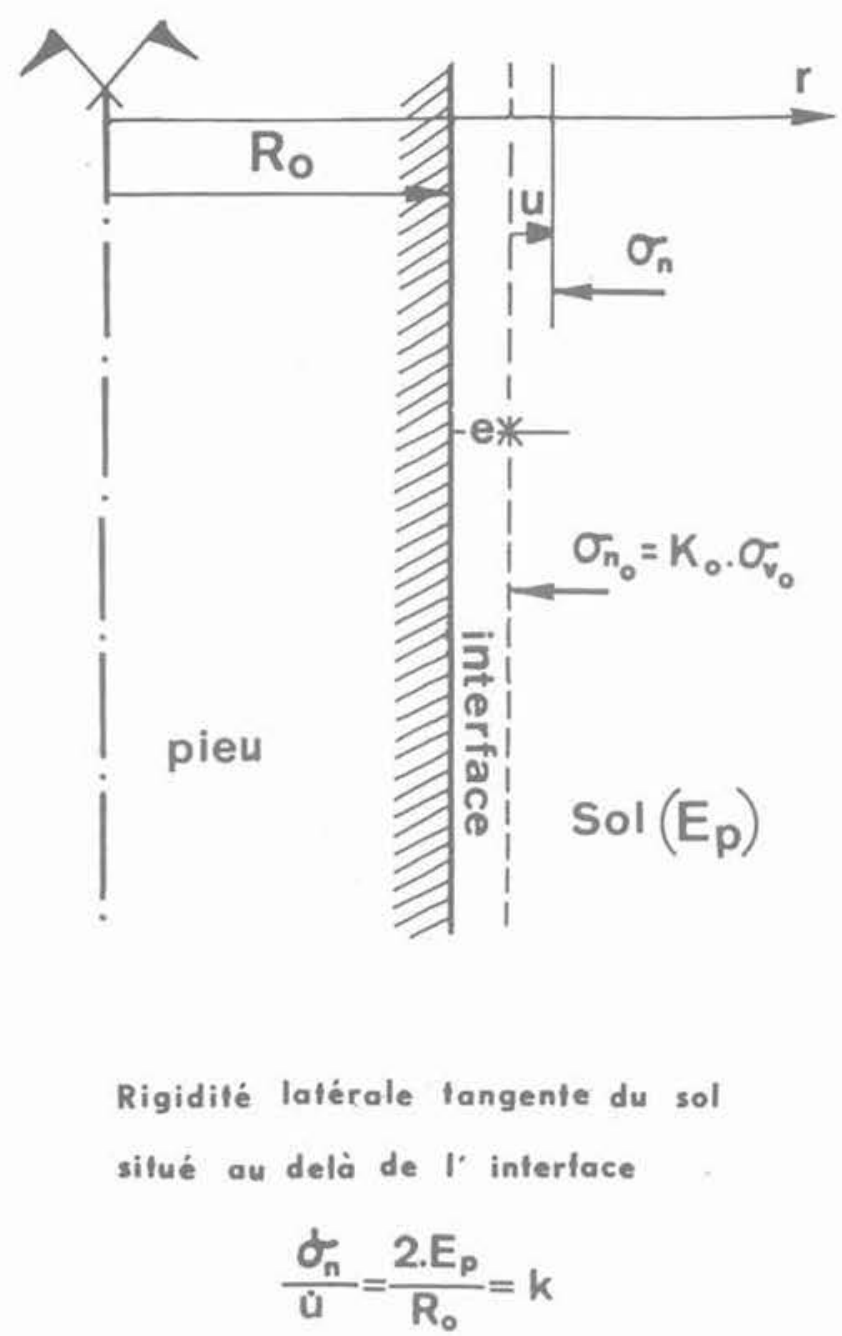

$$
\frac{\dot{\sigma}}{\dot{u}}=c t e=k
$$

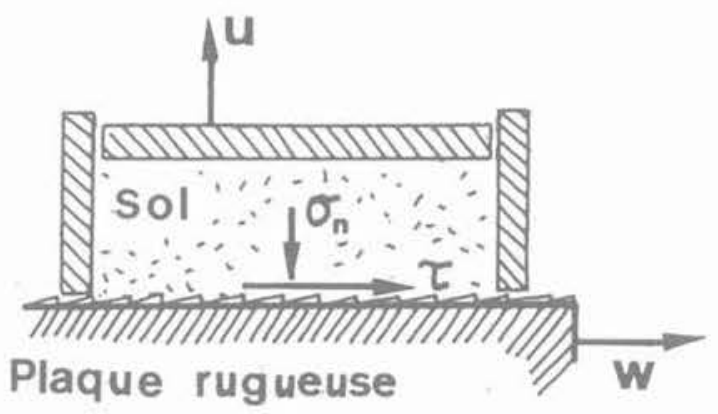

asservissement à $\mathrm{I}$ échantillon

$$
\frac{\delta_{n}}{u}=k
$$

Fig. 12. - Analogie entre cisaillement localisé du sol le long d'une inclusion sollicitée axialement et cisaillement direct à rigidité normale imposée par asservissement en Laboratoire.

ce qui définit la rigidité latérale tangente imposée par le sol (situé à $\mathrm{r}>\mathrm{R}_{\mathrm{o}}+\mathrm{e}+\mathrm{u}$ ) à l'interface durant le cisaillement localisé. De tels chemins de cisaillement peuvent être simulés à partir de la loi d'interface proposée précédemment et peuvent être réalisés en Laboratoire au prix d'une petite modification de la boîte de cisaillement classique ; il suffit pour cela de remplacer le poids générateur de $\sigma_{n}$ par un ressort de rigidité adéquate (fig. 13)

\subsubsection{Simulation de chemins de cisaillement à rigidité normale imposée, vérifications expérimentales}

Nous présentons ici trois intégrations numériques typiques de lois d'interface le long de chemins de cisaillement à rigidité imposée sur le sable $n^{\circ} 2$. Ces résultats ont été obtenus grâce à cent incréments de calcul correspondant chacun à un pas de déplacement tangentiel $\Delta w$ de $0,04 \mathrm{~mm}$. Le coefficient $\mathrm{x}$ caractéristique de l'interpolation a été pris égal à 1,1. Dans la mesure où les coordonnées $\xi$ et $\eta$ de la sollicitation ne sont pas connues à l'avance, des itérations sont nécessaires (PLYTAS (30)). L'incidence de la taille de la boite $(\varnothing=6 \mathrm{~cm}$ ou $\varnothing=10 \mathrm{~cm}$ ) apparaît nettement sur la figure 14, correspondant à $\gamma_{0}=16,70 \mathrm{kN} / \mathrm{m}^{3}$ (densité forte), $\sigma_{n 0}=1061 \mathrm{kPa}$ (contrainte normale initiale forte) et $\mathrm{k}=800 \mathrm{kPa} / \mathrm{mm}$ (rigidité faible). L'incidence d'une évaluation erronée de la densité initiale $\left(\gamma_{0} \pm 2 \%\right)$ ainsi que de la hauteur de l'échantillon sont mises en évidence sur les figures 15 et 16 . La comparaison des résultats des figures 15 et 16 montre également l'influence de la rigidité du sol environnant (c'està-dire du couple module pressiométrique, rayon de l'inclusion), puisque le cisaillement mobilisé est pratiquement doublé lorsque la rigidité est multipliée par 5. Les résultats expérimentaux figurant sur les figures 14. 15,16 sont dus à VALIN (35). 

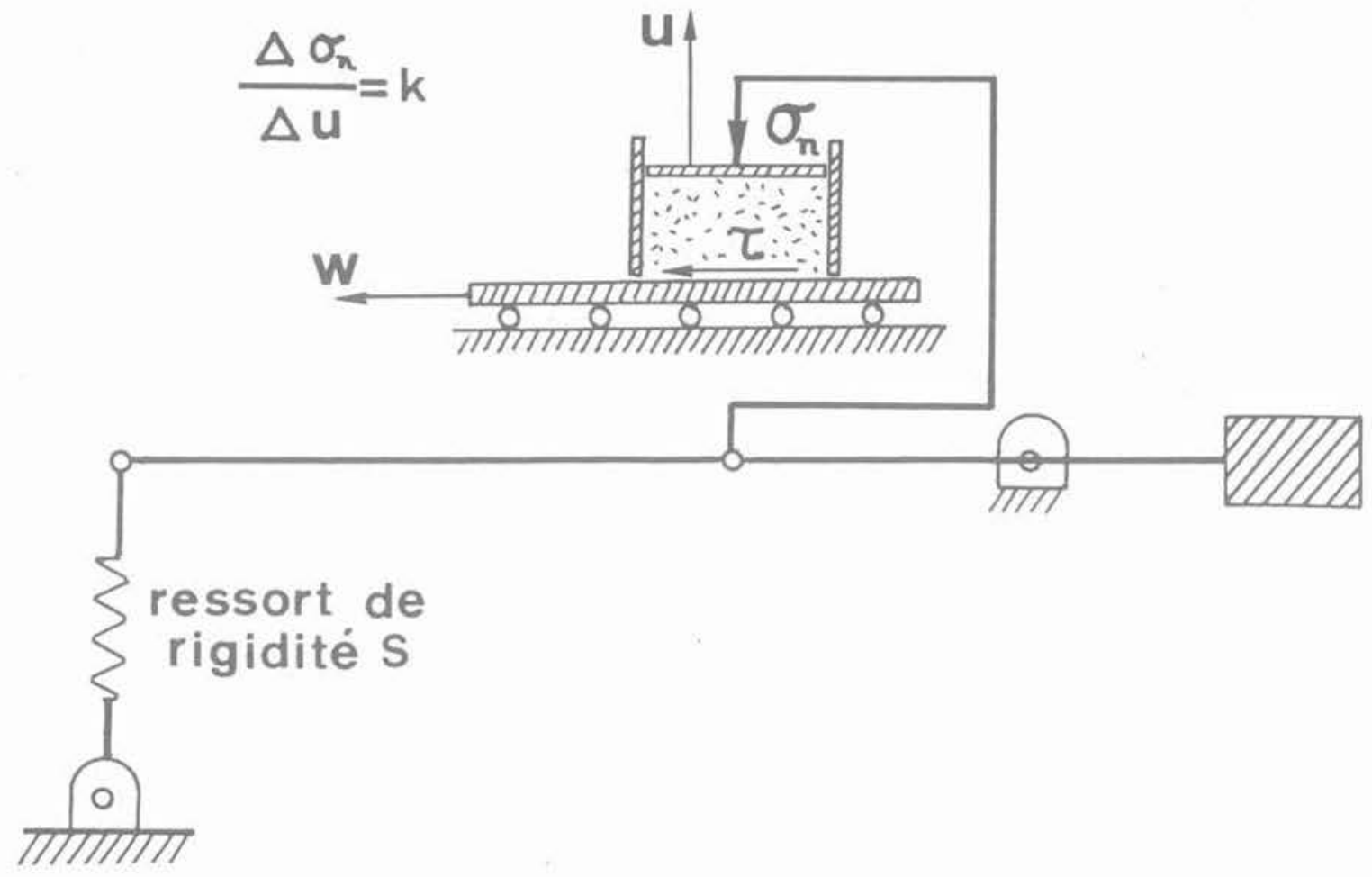

a) Schéma de principe.

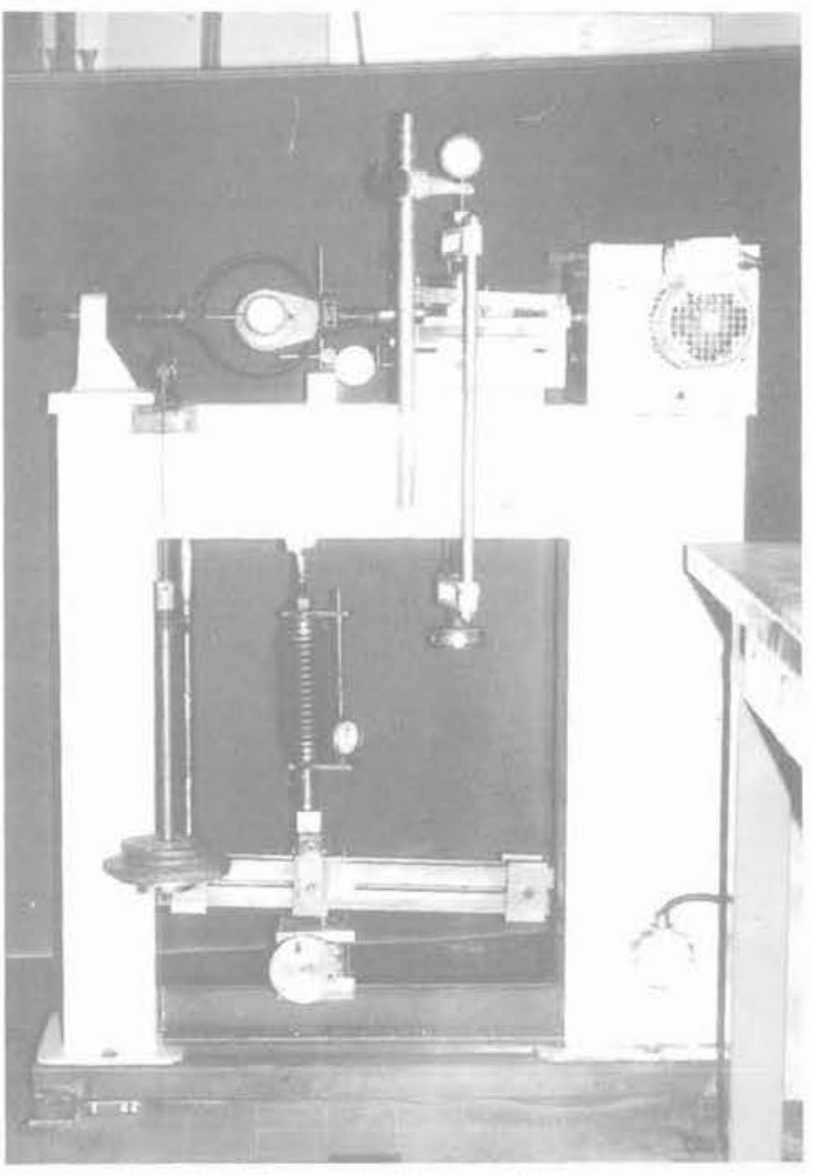

b) Photo de l'appareillage.

Fig. 13. - Appareillage de cisaillement direct à rigidité imposée.
3.2.3. Application à la prévision du coefficient $K_{\mathrm{lim}}$ de pression des terres

Nous avons exploité systématiquement les résultats de ces simulations quant aux paliers de $\sigma_{n}$ et $\tau$ atteints en fin de cisaillement, d'où une prévision possible du coefficient $\mathrm{K}_{\text {tim }}$ de pression des terres à saturation du frottement, fonction des paramètres $\gamma_{0}, \sigma_{n_{0}}$ et $\mathrm{k}$. Au prix d'une hypothèse sur le coefficient $\mathrm{K}_{\mathrm{o}}$ de pression des terres au repos, nous avons représenté $\mathrm{K}_{\text {tim }}$ en fonction de $\sigma_{v_{0}}$ (contrainte verticale initiale) et $\mathrm{k}$, pour deux densités initiales données. C'est l'objet des figures 17a et b, correspondant respectivement à une densité forte et faible du sable d'Hostun $n^{\circ} 2$. En plus des vérifications expérimentales possibles grâce aux essais de cisaillement à rigidité imposée de VALIN (35), nous avons comparé ces résultats à ceux de EISSAUTIER (14) obtenus lors d'arrachements de pieux modèles en chambre de calibration (ces essais ont d'ailleurs fourni des mesures de $\mathrm{K}_{0}$ ). La concordance des trois types d'approche est bonne, sauf à densité faible et à contrainte normale élevée. Il semblerait que l'évaluation de la densité initiale dans ces conditions en chambre de calibration (après montée en pression de la cuve) soit sujette à discussion. De toutes manières, il est clair que $\mathrm{K}_{\mathrm{lm}}$ décroît avec $\sigma_{\mathrm{v}}$, à rigidité $\mathrm{k}$ donnée; de plus, la simulation numérique indique que $\mathrm{K}_{\mathrm{lim}}$ pourrait même prendre des valeurs inférieures à $K_{0}$, pourvu que $\sigma_{v_{0}}$ soit suffisamment importante. Nous ne possédons que des vérifications expérimentales indirectes de ce résultat (BELOTTI et al. (10)), très plausible toutefois, qui apportent quelques éléments de réflexion sur le fonctionnement des pieux des travaux en haute mer, aussi bien que sur le problème de la mise en place par fonçage. 

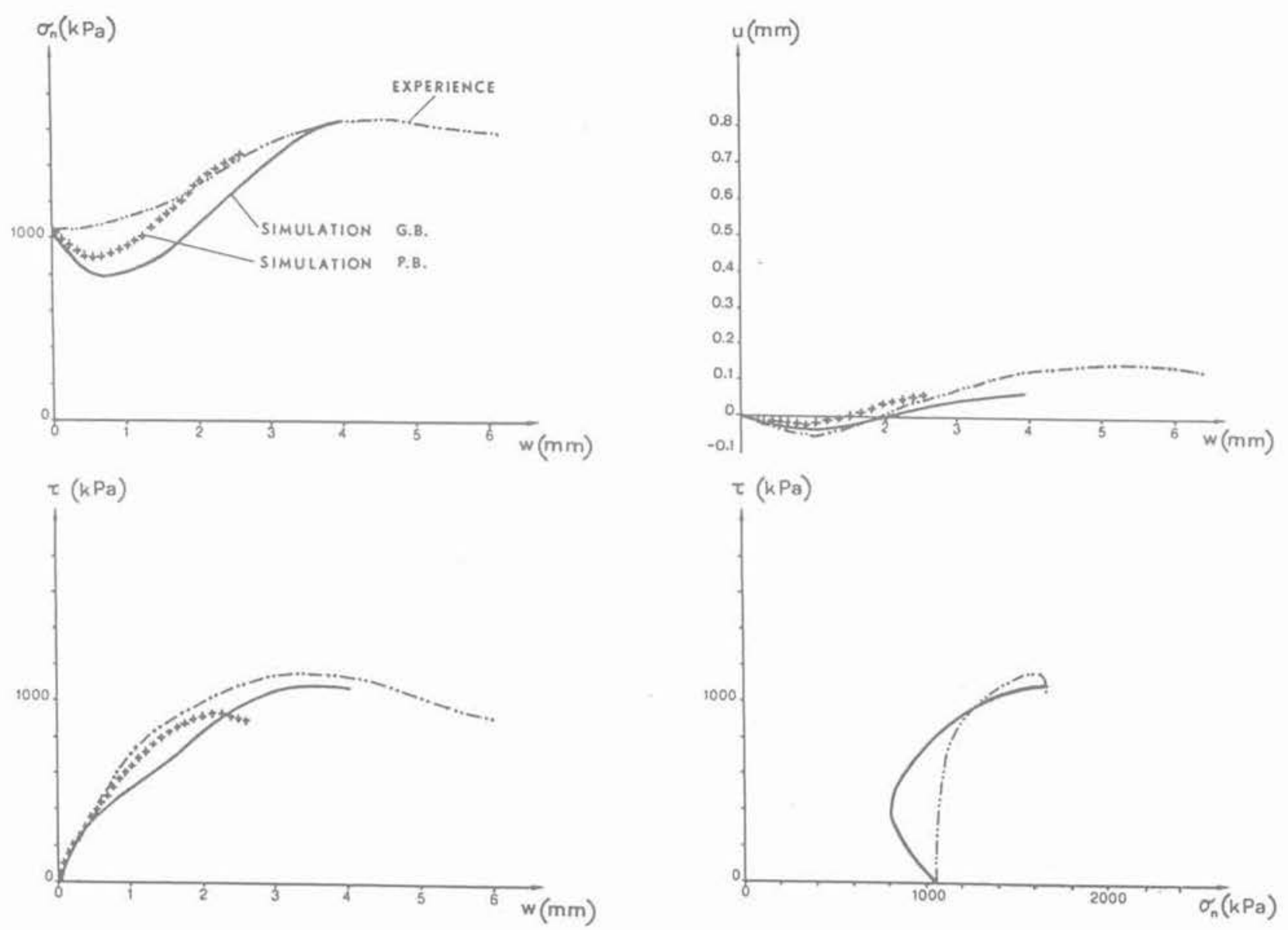

Fig. 14. - Chemin de cisaillement direct à rigidité imposée sur sable d'Hostun $n^{\circ} 2 ; \gamma_{0}=16,70 \mathrm{kN} / \mathrm{m}^{3}$ (densité forte); $\sigma_{n_{0}}=1061 \mathrm{kPa} ; \mathrm{k}=800 \mathrm{kPa} / \mathrm{mm}$ (rigidité faible). - Simulation numérique d'après PLYTAS (30) GB = Grande Boîte $(\varnothing=10 \mathrm{~cm}) \quad P B=$ Petite Boîte $(\varnothing=6 \mathrm{~cm})$ Essai d'après VALIN (35).

\section{CONCLUSION}

La bibliographie examinée, aussi bien que les essais et simulations présentés dans cette communication montrent que lorsqu'une inclusion ancrée dans le sol est sollicitée axialement. la contrainte normale à l'inclusion évolue durant le chargement; cette évolution peut être considérée comme une conséquence du couplage entre phénomènes tangentiels et normaux lors du cisaillement localisé au sein d'une fine couche de transition, désignée comme interface sol-inclusion. L'évolution des contraintes normales est peu importante le long des pieux courants de longueur moyenne, mais est manifeste le long des tirants ancrés à faible profondeur (dilatance) ainsi que le long des pieux très longs (contractance). Compte tenu de ce qui précède, on conçoit que l'essai de cisaillement direct classique, à contrainte normale constante ne puisse fournir tous les éléments de calcul du frottement latéral le long des inclusions moulées. Par contre, l'essai de cisaillement à rigidité normale imposée nous parait plus adapté à cette prévision, en tant que synthèse des propriétés au cisaillement de la fine couche de sol au contact du pieu, et des propriétés de compressibilité du reste du sol. De nombreuses connaissances relatives aux interfaces sol-inclusions manquent encore, notamment en ce qui concerne les sols fins, les sols calcaires et la pré- vision du frottement latéral le long des inclusions foncées et battues. Enfin, il nous paraît important de relativiser, mais aussi de dégager la signification des essais de Laboratoire et de leur simulation numérique : les essais de Laboratoire ne sont pas des modèles réduits, c'est-à-dire qu'il n'est pas possible de prévoir le comportement d'ouvrages réels à partir de ces essais et des règles de la similitude. Par contre, ce sont des essais de petite taille dont les paramètres sont généralement mieux contrôlés qu'in situ. A partir de l'instant où les mécanismes élémentaires sont établis pour des niveaux de contraintes couvrant le domaine relatif aux ouvrages réels, savoir simuler le comportement d'ouvrages de Laboratoire équivaut à savoir faire des calculs prévisionnels d'ouvrages réels, pourvu que les mesures indispensables existent.

\section{BIBLIOGRAPHIE}

1. R. BELLOTTI, G. BIZZI, V. GHIONNA, M. JAMIOLKOWSKI, S. MARCHETTI, E. PASQUALINI, Preliminary calibration tests of electrical cone and flat dilatometer in sand, design parameters in geotechnical engineering, B.G.S., London, 1979, vol. 2. 

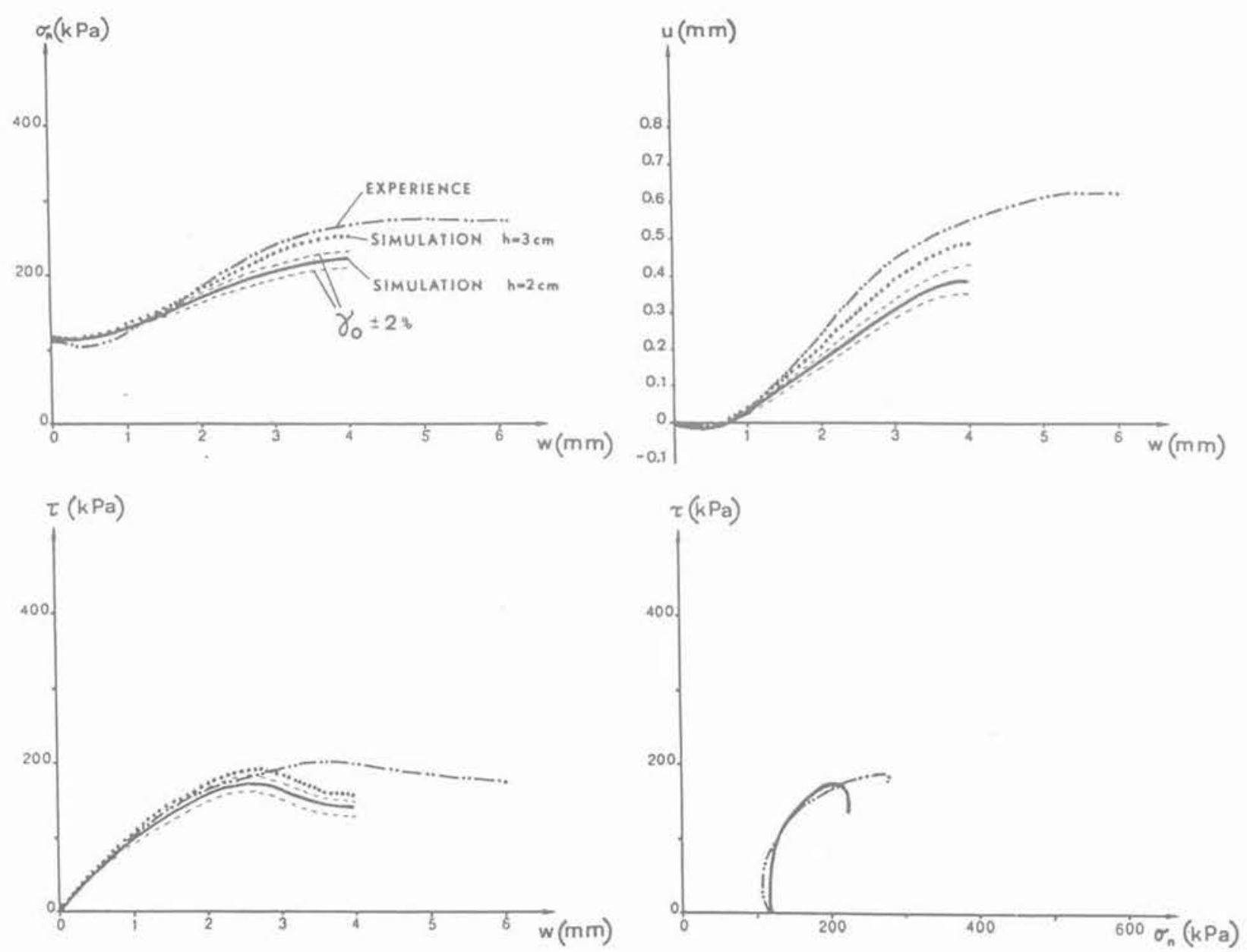

Fig. 15. - Chemin de cisaillement direct à rigidité imposée, sur sable d'Hostun $n^{\circ} 2 ; \gamma_{0}=16,90 \mathrm{kN} / \mathrm{m}^{3}$ (densité forte), $\sigma_{n_{0}}=122 \mathrm{kPa} ; k=266,7 \mathrm{kPa} / \mathrm{mm}$ (rigidité faible). d'après PLYTAS $(30) h=3 \mathrm{~cm} ; \quad h=2 \mathrm{~cm} ; \quad$ Essai d'après VALIN (35).

2. M. BOULON, R. CHAMBON, F. DARVE, P. FORAY, F. MORELOT, A. PUECH, A. ZINEBI. Tassements et force portante des pieux en milieu pulvérulent, Congrès Européen de Mécanique des sols, Vienne, Autriche, 1976.

3. M. BOULON, F. DARVE, J. DESRUES, P. FORAY, Soil structure coupling, nonlinéar rheological relationships and boundary conditions in soil mechanics, Int. Journal for Computers and Structures, vol. 9, pp. 293-303, 1978.

4. M. BOULON, J. DESRUES, P. FORAY, M. FORGUE, Numerical model for foundation under cyclic loading, application to piles, Int. Symposium on soils under cyclic and transient loading, Swansea, U.K., 1980.

5. M. BOULON, A. PUECH, Simulation numérique du comportement des pieux sous chargement axial cyclique. Revue Française de Géotechnique, $\mathrm{n}^{\circ} 26$, juin 1984

6. M. BOULON, Simulation numérique du comportement des pieux chargés axialement, thèse de Doctorat ès sciences, Université de Grenoble, à paraître, 1986.
7. M. BOULON, P. FORAY, C. PLYTAS, Soilstructure interface behaviour and skin friction, Geotechnique symposium; in print. The Engineering Application of Direct and Simple Shear Testing, à paraître, 1986.

8. H.M. COYLE, R.R. CASTELLO, New design correlations for piles in sand, Journ. of the Geotechnical Engineering division, A.S.C.E., vol. 107, G.T.7, pp. 965-985, 1981.

9. F. DARVE, Une formulation incrémentale des lois rhéologiques, application aux sols, thèse de Doctorat ès sciences, Université de Grenoble, 1978.

10. A.G. DAVIS, C. PLUMELLE, Comportement des tirants d'ancrage dans un sable fin, Revue Française de Géotechnique, $n^{\circ} 10,1979$.

11. C.S. DESAI, Numerical design analysis for piles in sand, Journal of the Geotechnical Engineering division, A.S.C.E., G.T.6, vol. 100, pp. 613 . $635,1974$.

12. DESAI, Behaviour of interfaces between structural and geologic media, Int. Conf. on recent advan- 

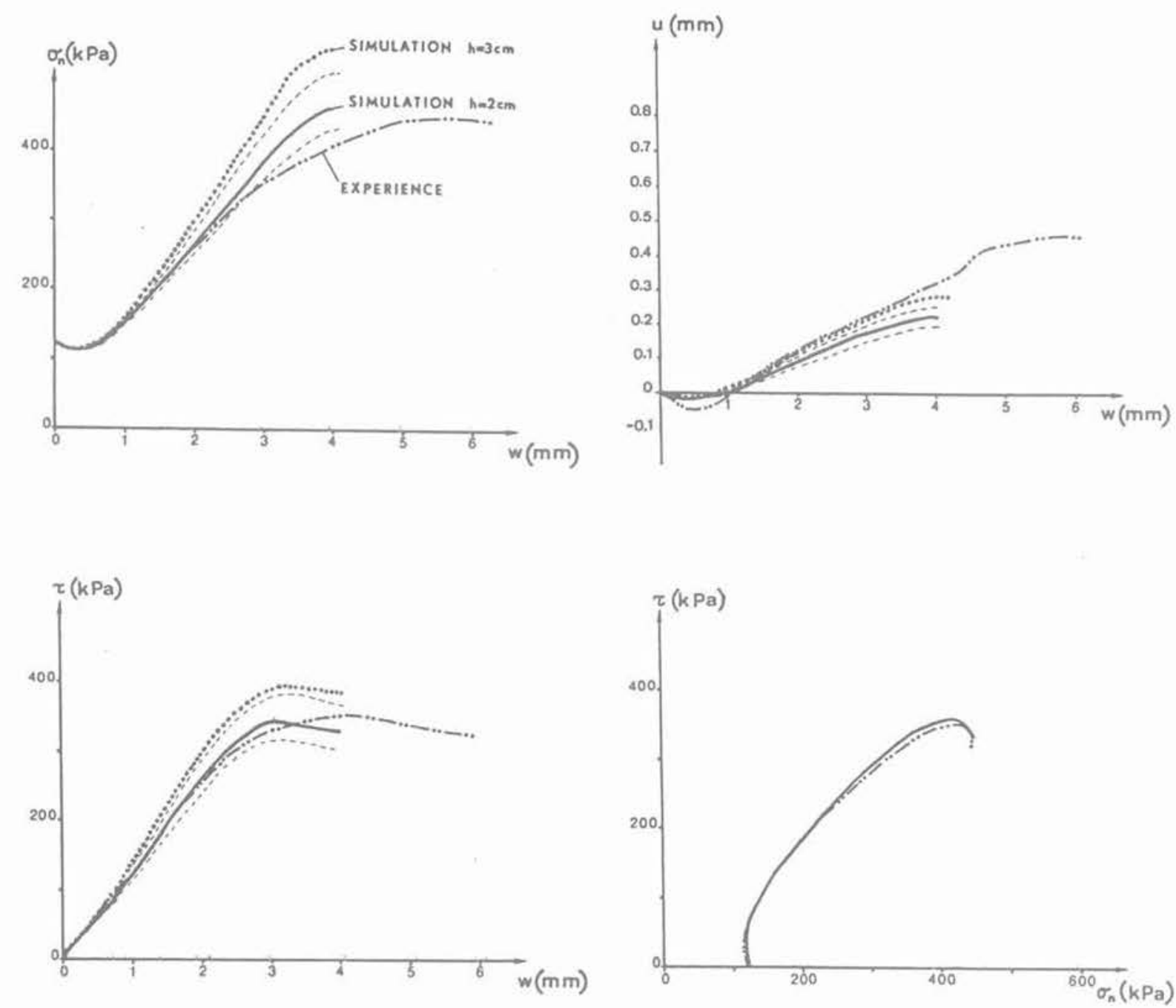

Fig. 16. - Chemin de cisaillement direct à rigidité imposée, sur sable d'Hostun $n^{\circ} 2 ; \gamma_{0}=16,8 \mathrm{kN} / \mathrm{m}^{3}$ (densité forte), $\sigma_{n_{0}}=122 \mathrm{kPa} ; \mathrm{k}=1600 \mathrm{kPa} / \mathrm{mm}$ (rigidité forte). Simulation numérique d'après PLYTAS (30) $h=3 \mathrm{~cm} ; \quad h=2 \mathrm{~cm} ; \quad$ Essai d'après VALIN (35).

ces in Geotechnical earthquake engineering and soil dynamics, Saint-Louis, U.S.A., 1981.

13. C.S. DESAI, Constitutive Modelling and soilstructure interaction, Numerical Methods in Geomechanics, ed. by J.B. MARTINS, proc. of the NATO advanced study Institute, Vimeiro, Portugal, 1981, pp. 103-143, Reidel publishing Company, Dordrecht, 1982.

14. M. EISSAUTIER, Frottement latéral des pieux en milieu pulvérulent; essais en chambre de Calibration, Thèse de $3^{e}$ cycle, Université Scientifique et Médicale, Grenoble, et Institut National Polytechnique de Grenoble, Grenoble, 1986.

15. J. FEDA, Skin friction of piles, Congrès Européen de Mécanique des sols, Vienne, 1976, pp. 423428.

16. J. FEDA, Mechanics of particulate materials. The principles. Elsevier ed. Amsterdam, 1982.

17. E. FLAVIGNY, P. FORAY, F. DARVE, Prediction of undrained behaviour of sands, discussion A.S.C.E., G.T.9, vol. 105, September 1979.
18. R. FRANK, Étude théorique du comportement des pieux sous charge verticale: introduction de la dilatance, Thèse de Docteur Ingénieur, université Paris Vl, 1974.

19. R. FRANK, A. GUENOT, P. HUMBERT, Numerical analysis of contacts in geomechanics, proc. of the fourth Int. Conf. on numerical methods in Geomechanics, Edmonton, Canada, 1982.

20. J.C. GABAIX, M. BUSTAMANTE, D. GOUVENOT, Essais de pieux scellés par injection sous pression, Annales de l'I.T.B.T.P., série sols et fondations, $n^{\circ} 331$, septembre 1975 .

21. J. GHABOUSSI, E.L. WILSON, J. ISENBERG. Finite element for rock joints and interfaces, Journ. of the soil mechanics and foundations division, A.S.C.E., S.M.10, vol. 99, pp. 833-848, 1973.

22. R.E. GOODMAN, R.L. TAYLOR, T.L. BREKKE, A model for the mechanics of jointed rock, Journ. of the soil mechanics and foundations division, A.S.C.E., S.M.3, vol. 94, pp. 637-659, 1968. 

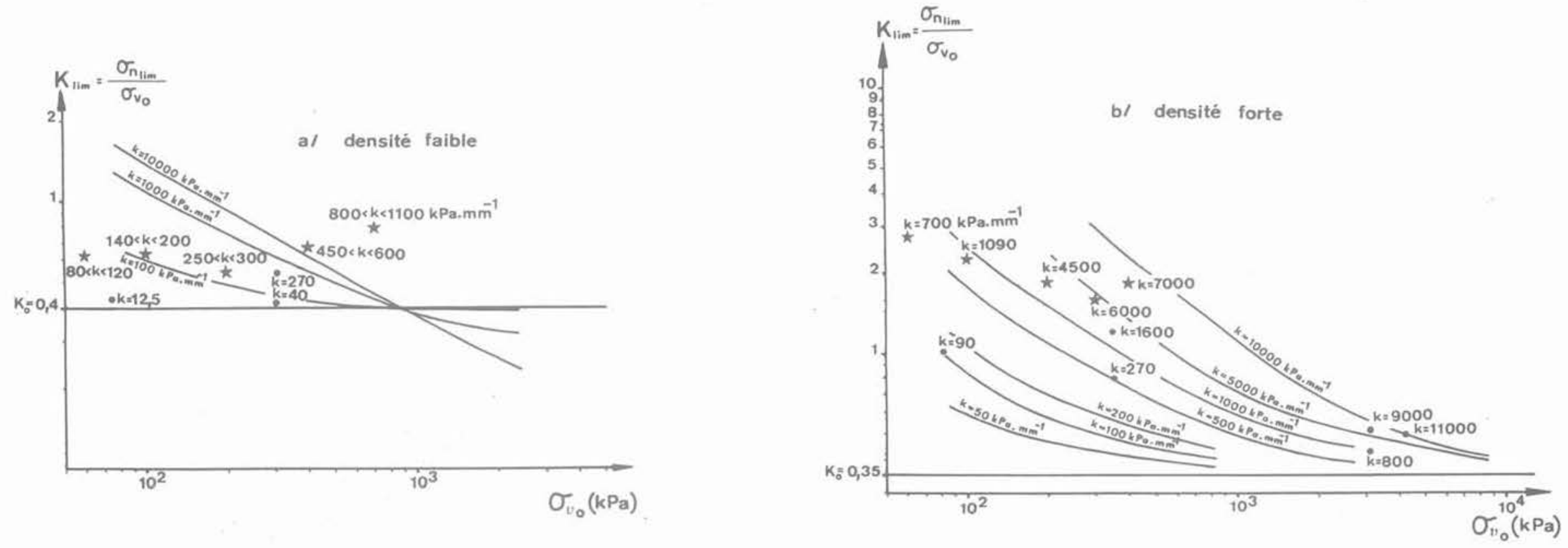

Fig. 17. - Coefficient de pression $K$ en fonction de la contrainte verticale initiale $\sigma_{v_{0}}$ et de la rigidité du milieu environnant $k=\frac{2 . E_{\rho}}{R} ; \quad$ simulation d'après PLYTAS (30);

- Essais d'après VALIN (35); * Essais d'après EISSAUTIER (14). 
23. R.E. GOODMAN, J. DUBOIS, Duplication of dilatancy in analysis of jointed rocks, Journ. of the soil mechanics and foundations division. A.S.C.E., S.M.4, vol. 98, pp. 399-422, 1972.

24. F.E. HEUZE, R.E. GOODMAN, A. BORNSTEIN, Numerical analysis of deformability tests in jointed rock - "joint pertubation and no tension". finite element solutions, Rock mechanics, $n^{\circ} 3$, pp. 13-24, 1971.

25. F.E. HEUZE, T.G. BARBOUR, New models for rock joints and interfaces, Journal of the Geotechnical Engineering division, A.S.C.E., G.T.5, vol. 108 , pp. $757-776,1982$.

26. D.M. HOLLOWAY, G. WAYNE CLOUGH, A.S. VESIC, The mechanics of pile soil interaction in cohesionless soils, research report, Duke University, Durham, 1975.

27. S. LABANIEH, Modélisations non linéaires de la rhéologie des sables et applications. Thèse de Doctorat ès sciences, Université Scientifique et Médicale de Grenoble, Institut National Polytechnique de Grenoble, novembre 1984.

28. W. LEICHNITZ, Mechanische Eigenschaften von Felstrenn-flächen in direkten Scherversuch, Dissertation, Karlsruhe, 1981.

29. H. OSTERMAYER, F. SCHEELE, Research on ground anchors in non-cohesive soils, Revue Française de Géotechnique, n³, 1978.

30. C. PLYTAS, Contribution à l'étude expérimentale et numérique des interfaces sols granulaires-structures: application à la prévision du frottement latéral des pieux. Thèse de Docteur Ingénieur, Université Scientifique et Médicale de Grenoble. octobre 1985.
31. A. PUECH, P. FORAY, M. BOULON, J. DESRUES, Calcul des pieux à larrachement à partir d'un modèle numérique en contraintes effectives. premiers résultats, Congrès Européen de Mécanique des sols, Brighton, septembre 1979.

32. A. PUECH, Basic data for the design of tension piles in silty Soils. III ${ }^{\mathrm{d}}$ Conf. on the behaviour of offshore structures, Boston, U.S.A., 1982.

33. A. PUECH, M. BOULON, Y. MEIMON, Behaviour of tension piles: field data and numerical modelling, second Int. Conf. on numerical methods in offshore piling, Austin, U.S.A., 1982.

34. F. SCHLOSSER, A. GUILLOUX, Le frottement dans le renforcement des sols, Revue Française de Géotechnique, n 16, août 1981.

35. M. VALIN, Étude expérimentale des interfaces sol-pieu : essais de cisaillement direct à rigidité imposée. Mémoire de DEA, Institut National Polytechnique, Grenoble, septembre 1985.

36. E. WERNICK, Stresses and strains on the surface of anchors, Revue Française de Géotechnique, $\mathrm{n}^{\circ} 3,1978$.

37. Y. YOSHIMI, T. KISHIDA, A ring torsion apparatus for evaluating friction between soil and metal surfaces, Geotechnical Testing Journal G.T.J.O.D.J., vol. 4, $n^{\circ}$ 4, December 1981 , pp. 145-152.

38. O.C. ZIENKIEWICZ, B. BEST, C. DULLAGE, K.G. STAGG, Analusis of non linear problems in rock mechanics with particular reference to jointed rock systems, Proc. of the $2^{\text {nd }}$ cong. of the Int. Soc. for Rock Mechanics, Belgrade, Yugoslavia, 1970. 